\title{
Whole-body modelling of people from multi-view images to populate virtual worlds
}

\author{
Hilton,A., Beresford,D., Gentils,T., Smith,R.J., Sun,W. and Illingworth,J. \\ Centre for Vision, Speech and Signal Processing \\ University of Surrey, Guildford GU25XH, UK \\ a.hilton@surrey.ac.uk
}

http://www.ee.surrey.ac.uk/Research/VSSP/3DVision/VirtualPeople

\begin{abstract}
In this paper a new technique is introduced for automatically building recognisable moving 3D models of individual people. A set of multi-view colour images of a person are captured from the front, side and back using one or more cameras. Model-based reconstruction of shape from silhouettes is used to transform a standard 3D generic humanoid model to approximate the persons shape and anatomical structure. Realistic appearance is achieved by colour texture mapping from the multi-view images. Results demonstrate the reconstruction of a realistic 3D facsimile of the person suitable for animation in a virtual world. The system is low-cost and is reliable for large variations in shape, size and clothing. This is the first approach to achieve realistic model capture for clothed people and automatic reconstruction of animated models. A commercial system based on this approach has recently been used to capture thousands of models of the general public.
\end{abstract}

Keywords: Avatar, Virtual Human, Whole-body Modelling, 3D Vision, VRML 
There is increasing demand for a low-cost system to capture both human shape and appearance. Potential applications for such a system include population of virtual environments, communication, multi-media games and clothing. This paper presents a technique for capturing recognisable models of individual people for use in VR applications. For instance each participant in a multi-user virtual environment could be represented to others as an 'avatar' which is a realistic facsimile of the persons shape, size and appearance. The key requirements for building models of individuals for use in virtual worlds are:

- Realistic models

- Animatable movements

- Low-cost (automatic) acquisition

These requirements contrast with previous objectives of whole-body measurement systems which were principally designed to obtain accurate metric information of human shape. Such systems typically capture low-resolution colour and have restrictions on surface properties which result in no measurements for areas of dark colours and hair. Current wholebody measurement systems are highly expensive and require expert knowledge to interpret the data and build animated models [Paquette, 1996]. These systems are suitable for capturing measurements of individual people for clothing applications but are not capable of capturing recognisable models for VR or photo-realistic models for computer animation. Recent research has addressed reconstructing realistic animated face models [Akimoto et al., 1993, Escher and Magnenat-Thalmann, 1997, Fua and Miccio, 2000, Lee and Magnenat-Thalmann, 1998] and whole-body models of kinematic structure [Plankers et al., 1999, Kakadiaris and Metaxas, 1998a] from captured images. The objective of this research is to extend this work to address the reconstruction of whole-body models of shape and appearance from captured images.

In this paper we introduce a technique for building models of individual people from a set of four orthogonal view images using standard camera technology. The reconstruction from multiple orthogonal view images is analogous to previous work on facial modelling [Akimoto et al., 1993, Lee and Magnenat-Thalmann, 1998, Mortlock et al., 1997]. A major feature of our approach is that we can reconstruct recognisable colour models of people who are fully clothed. The aim 
is to capture accurate appearance together with approximate shape information and not to accurately measure the underlying body dimensions. The reconstruction algorithm modifies a generic humanoid model to fit the silhouette shape of a particular individual from multiple view images. This approach follows the 'functional modelling' paradigm [Terzopoulos, 1994] of modifying the shape and appearance of a generic model containing the kinematic structure to generate a model which can be animated. This research uses a generic model based on the VRML Humanoid Animation H-Anim[Roehl, 1997] standard. The resulting model of a specific person is in a standard format which can be viewed in any VRML97 compliant internet browser to obtain a recognisable moving virtual model (avatar) of a particular individual. It is envisaged that the commercial availability of low-cost whole-body capture will open up a mass market for personalised plug-ins to multimedia and games packages with individuals having a personal set of models.

\section{Previous Work}

Capturing of human shape and appearance has received considerable interest throughout history. This section attempts to summarise the principal achievements of recent related research in reconstructing realistic models of people using active 3D surface measurements systems and passive 2D images. Modelling facial appearance and expression has been extensively addressed as this is the most important attribute for person-to-person communication. Whole-body capture initially addressed measurements systems for apparel design and ergonomics. Only recently have whole-body capture systems begun to address modelling of an individuals appearance and characteristic gestures.

\subsection{Object Modelling}

Our approach integrates two areas of computer vision research 'model-based fitting' and 'silhouette based shape reconstruction' to produce a powerful new method of 'model-based 3D shape reconstruction'. This enables reconstruction of the approximate 3D shape of a specific person from a set of two or more orthogonal view silhouette images.

Silhouette based reconstruction of 3D shape has received considerable interest for capturing object models [Szeliski, 1990, Niem and Wingebermuhle, 1997, Fitzgibbon et al., 1998]. Re- 
cent research has resulted in the reconstruction of $3 \mathrm{D}$ volumetric models of moving objects from sequences of silhouette images captured using multiple cameras from different views [Moezzi et al., 1997, Kanade and Rander, 1997]. Niem [Niem and Wingebermuhle, 1997] has demonstrated that highly realistic object models can be captured by taking a large number of images from different views of an object against a uniform blue background. Camera viewpoint and intrinsic parameters are computed for each image based on a static calibration pattern within the scene. Fitzgibbon et al. [Fitzgibbon et al., 1998] have recently demonstrated silhouette based reconstruction using auto-calibration techniques based on tracking points on the object surface without the need for a static calibration pattern. Combination of the extracted silhouette images into a 3D volumetric framework using projective geometry enables coarse approximation of the object surface shape. Colour texture mapping of the approximate shape model achieves highly realistic object models suitable for immersive virtual environments. An inherent limitation of silhouette based approaches is that concave surface shapes can not be reconstructed. The passive approach to $3 \mathrm{D}$ shape reconstruction does not achieve the metric accuracy of active measurement systems. However, the passive approach achieves a greater level of photo-realism in reconstructing fine surface detail.

In this paper we combine silhouette based 3D shape reconstruction with model-based fitting techniques to obtain an approximation of the shape of an individual person. The use of a model-based approach allows reconstruction from a minimal set of two orthogonal images and enables approximation of concave surface shapes. Terzopoulos [Terzopoulos et al., 1988] introduced fitting of deformable models to image silhouettes to recover shape and non-rigid motion. Model-based techniques have been widely used for fitting 3D measurements of surface shape using deformable meshes [Hilton and Goncalves, 1995], parametric deformable models [McInerney and Terzopoulos, 1996] and super-quadrics [Lejeune and Ferrie, 1996]. However, model-based shape fitting fails to accurately model fine surface detail and is limited to the reconstruction of objects with smooth surfaces. No results have been demonstrated for accurate reconstruction of highly complex objects with large shape variations such as the human body.

\subsection{Head Modelling}

There is a considerable body of literature addressing the goal of realistic modelling of the head and face of individual people. In modelling a particular person the face is the most important 
component which must be modelled realistically to achieve an acceptable representation. Fully automatic acquisition of realistic animated 'talking-head' models of individual people remains an open-problem. The principal difficulty in fully automated model acquisition is the considerable variability in shape and appearance between individuals shape and hair. Recent research has begun to address the problem of realistic modelling of facial expression.

Techniques have been presented [Akimoto et al., 1993, Fua and Miccio, 2000, Kurihara and Kiyoshi, Lee and Magnenat-Thalmann, 1998, Mortlock et al., 1997, Sannier and Magnenat Thalmann, 1997] which use captured 2D images to modify the shape of a 3D generic face model to approximate a particular individual. Photogrammetric techniques are used to estimate the 3D displacement of points on the surface of a generic model from multiple camera images. Texture mapping of the captured images is then used to achieve a recognisable 3D face model. Facial features from the captured images are used to register them with corresponding 3D points on the generic model. Correct registration of feature points is required to ensure proper animation of the resulting model. Initial work [Kurihara and Kiyoshi, 1990] manually labelled feature points on several view images. Automatic feature labelling with limited manual intervention to correct mistakes has been achieved in recent work [Mortlock et al., 1997, Lee and Magnenat-Thalmann, 1998]. This approach has also been extended to model individual facial expressions [Guenter et al., 1998, Pighin et al., 1998]. Reconstruction of facial shape from two orthogonal view images has been demonstrated using silhouette template fitting [Akimoto et al., 1993] and feature point labelling [Mortlock et al., 1997, Lee and Magnenat-Thalmann, 1998]. The use of orthogonal view images is analogous to the approach to whole-body reconstruction presented in this paper.

Reconstruction of animated face models for individual people from dense 3D surface measurements has been demonstrated [Escher and Magnenat-Thalmann, 1997, Lee et al., 1995, Terzopoulos, using data from active 3D range sensors. A generic face model is adapted to the 3D measurement points to accurately model the face shape for animation. Colour information available from the range sensor is mapped on to the face. This approach achieves highly accurate modelling of the face shape but lower quality visual appearance than image based techniques. More recent research [Fua and Miccio, 2000, Fua, 1998] has reconstructed dense 3D measurements from stereo images and video sequences of the face. A least-squares surface fitting approach is then used to fit a generic face model to the dense depth map with constraints for feature point location and silhouette shape. Currently feature points are specified manually. This image 
based approach achieves highly realistic face models of individual people suitable for animation. DeCarlo and Metaxas [DeCarlo and Metaxas, 1996, DeCarlo and Metaxas, 1999] used optical flow to fit constrained deformable models to video sequences for estimation of both shape and movement of the face. Anthropometric constraints have been introduced to model the variation in head shape for generating synthetic face models [DeCarlo et al., 1998]. Recent work [Blanz and Vetter, 1999] achieved photo-realistic facial reconstruction based on a statistical model of the variation in face shape and appearance derived from a database of captured 3D colour head models.

Face modelling techniques using multiple view images are similar to the approach presented in this paper for whole-body modelling. A difference in our approach is the use of silhouette data to register the images with a generic model and estimate the 3D shape. Silhouette shape is used to reliably locate feature points on images of a clothed person with large variation in size, shape and appearance. A current limitation of the automatic whole-body modelling using silhouette shape is the reliability of facial reconstruction. Techniques for facial modelling [Blanz and Vetter, 1999, Fua and Miccio, 2000, Lee and Magnenat-Thalmann, 1998, Mortlock et al., 199 could be used in conjunction with whole-body reconstruction to achieve improved facial modelling. However, current image based techniques for face modelling require a full resolution image to enable automatic feature labelling. In addition, current face modelling techniques may fail to reliably reconstruct face shape automatically for large variations in shape and appearance due to hair, glasses and beards.

\subsection{Whole-body Modelling}

There is considerable interest in capturing models of individual people for anthropometric studies, clothing design, human factors analysis, teleconferencing, and virtual reality. A number of systems have been developed for measurement of human shape by active projection of a light stripe or pattern onto the subject and triangulation of the image to obtain dense 3D surface measurements. Several 'whole body' measurement systems are now commercially available based on laser stripe triangulation including Cyberware, Hamamatsu and Vitronic, for a recent review see [Paquette, 1996]. Systems are capable of capturing dense 3D surface measurements with an accuracy of approximately $2-3 \mathrm{~mm}$ and capture times of $10-20 \mathrm{sec}-$ onds. However, such devices are highly expensive and therefore are only available for spe- 
cialist anthropometric studies such as military clothing or large scale surveys. Identification of anatomical landmarks from surface measurement data is a primary goal of current research [Dekker et al., 1998, Geisen et al., 1997]. The authors are not aware of any reported technique for automatically generating animated whole-body models from 3D scanned data. A principal limitation of current whole-body measurement systems for capturing realistic models of individual people is the quality of the captured colour information. Current systems are designed primarily for accurate measurement of surface shape and do not provide photo-realistic colour.

The model-based reconstruction algorithm presented in this work addresses modelling people from a set of colour images. Previous research has achieved reconstruction of the 3D shape, appearance and movement of a person from multi-view video sequences using reconstruction from a forty camera stereo setup [Kanade and Rander, 1997] and an eight camera silhouette system [Moezzi et al., 1997]. These systems address the long-term aim of non-invasive capture of 3D video. However, current systems require expensive hardware and do not address reconstruction of a model of a particular person. Recently a twelve camera multi-view system for whole-body measurement was presented [Gu et al., 1998] which enables capture of the shape of a particular person in a single pose. The shape is reconstructed without the use of a generic humanoid model by fitting parametric super-quadric models to the silhouette image for each view. This system results in a model of the $3 \mathrm{D}$ shape of a particular individual which is of sufficient accuracy to provide dimensional measurements for clothing design.

Modelling of human shape and kinematic structure has been addressed for captured images sequences [Hilton et al., 1999, Plankers et al., 1999, Kakadiaris and Metaxas, 1998a, Wingbermuhle and A multiple pose approach is introduced in [Kakadiaris and Metaxas, 1998a] for performing a spatio-temporal analysis of a captured human silhouette sequence to determine the joint positions and 3D shape of body parts. Multi-view image sequences are used to animate a customised virtual human model [Kakadiaris and Metaxas, 1998b]. Results demonstrated the segmentation of the body into parts based on a side view of the person performing a pre-defined sequence of arm and leg movements. Reconstruction of models of body-parts using a stereo video to estimate the 3D surface shape has been demonstrated [Plankers et al., 1999]. A least squares approach is used to simultaneously estimate the shape and kinematic structure. Initial results demonstrate the estimation of shape and kinematic structure of the arm from a frontal image sequence. Model-based reconstruction of upper-body shape from video sequences 
[Wingbermuhle and Weik, 1997] has been demonstrated by fitting a simple generic humanoid model to the silhouette of a particular person in each image frame.

Unlike previous whole-body modelling techniques the approach presented in this paper aims to reconstruct a recognisable model of a persons shape and appearance. The captured silhouette images of a person in a single pose are used to modify the shape of a generic humanoid model to approximate the shape of a particular individual and estimate their kinematic structure. Techniques for estimating kinematic structure from image sequences [Kakadiaris and Metaxas, 1998a, Plankers et al., 1999] could be combined with the current approach to accurately estimate joint positions using images of a person in multiple poses. This would significantly improve the accuracy of the reconstructed kinematic structure for large variations in shape, size and clothing.

\section{Overview}

An overview of the model-based 3D human shape reconstruction algorithm is illustrated in Figure 1. A generic 3D humanoid model is used as the basis for reconstruction as shown in Figure 1(a). Four synthetic images are generated for orthogonal views (front,left,right, back) of the model by projection of the generic model as illustrated in Figure 1(b). To reconstruct a model of a person four orthogonal view images are captured with the subject in approximately the same posture as the generic model. This is illustrated in Figure 1(c). We will refer to captured images of a particular person as the 'data images' and to images of the generic 3D model as the 'model images'.

Silhouette extraction is performed on the model and data images and a small set of key feature points are extracted as illustrated in Figure 1(d) and (e). Initial alignment of the feature points between the model and data ensures that separate functional body parts of the generic model (arms, legs and head) are correctly mapped to corresponding parts of the captured image silhouettes. Correct correspondence of body parts is required to achieve correct animation of the reconstructed 3D model of a particular person. A 2D-to-2D linear affine mapping between the model and data image silhouettes is introduced to establish a dense correspondence for any point inside the silhouette. This correspondence can be used to map the colour information from the data image onto the model image as illustrated in Figure 1 (f).

The dense 2D-to-2D mapping for a single image is used to define the shape deformation 
of the $3 \mathrm{D}$ model in a plane orthogonal to the view direction. Applying this deformation to the 3D generic model achieves a 2D-to-3D linear mapping of the image silhouette shape onto the shape of the 3D model. This model-based 2D-to-3D mapping is the core of the technique for reconstruction of 3D models of people. Integrating shape deformation information from two or more orthogonal views gives three orthogonal components of shape deformation. Applying this deformation to the generic model we can approximate the shape of a particular individual as illustrated in Figure 1(g). Combining the 3D shape with the 2D-to-2D mapping of the colour information we can obtain a colour texture mapped 3D model as illustrated in Figure 1(i). The resulting reconstructed 3D model provides a realistic representation of a particular individual. The articulated joint structure of the generic functional model can then be used to generate movement sequences for a particular individual in a virtual world. Walking and jumping animations derived from motion capture data are illustrated in Figures 1(h) and (j).

\section{Model-based avatar reconstruction}

This section introduces in detail each stage in the reconstruction of an articulated model of a specific person from multi-view images. The algorithm automatically reconstructs a model of the shape and appearance of a particular individual without any user intervention. This approach addresses whole-body modelling and uses the same algorithm for all body parts. This could be combined with specific algorithms for reconstructing the face [Lee and Magnenat-Thalmann, 1998, Mortlock et al., 1997] to obtain realistic animated face models.

\subsection{Generic human model specification}

Definition of a standard 3D humanoid model has recently received considerable interest for both efficient coding [Koenen, 1996] and animation in virtual worlds [Roehl, 1997]. In this work we have adopted the draft specification of the VRML Humanoid Animation Working Group (HAnim) which defines a humanoid model structure which can be viewed using any VRML97 compliant browser. A set of 3D humanoid models based on the draft standard are publicly available from the humanoid animation web site [Roehl, 1997]. The generic humanoid model used in this work is shown in Figure 2. The H-Anim draft standard defines a hierarchical articulated joint structure to represent the degrees-of-freedom of a humanoid. The humanoid shape 


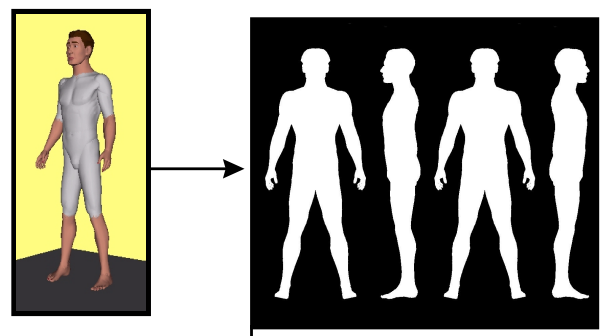

(a) Generic model (b) Model projection

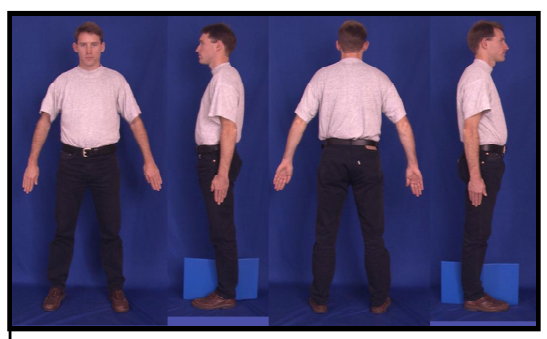

(c) Captured images

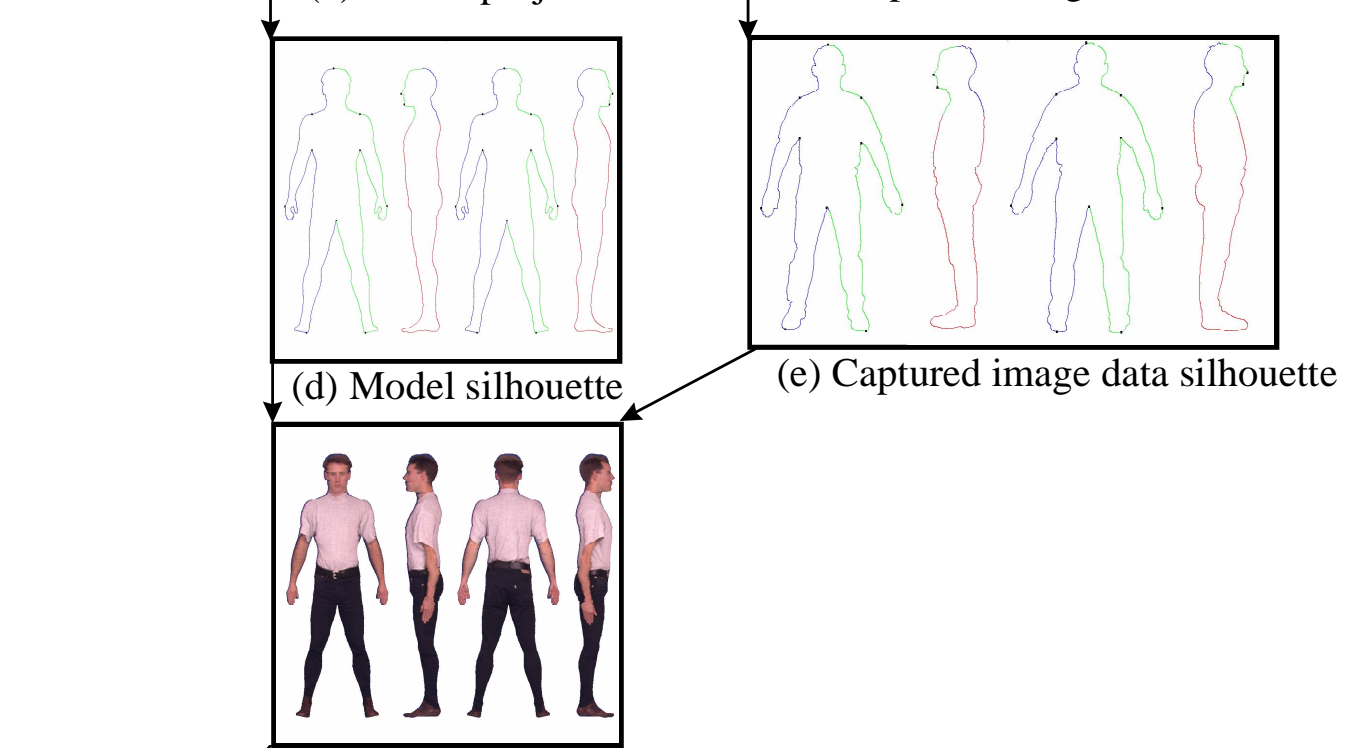

(f) Dence $2 d$ mapping of data image on model silhouette

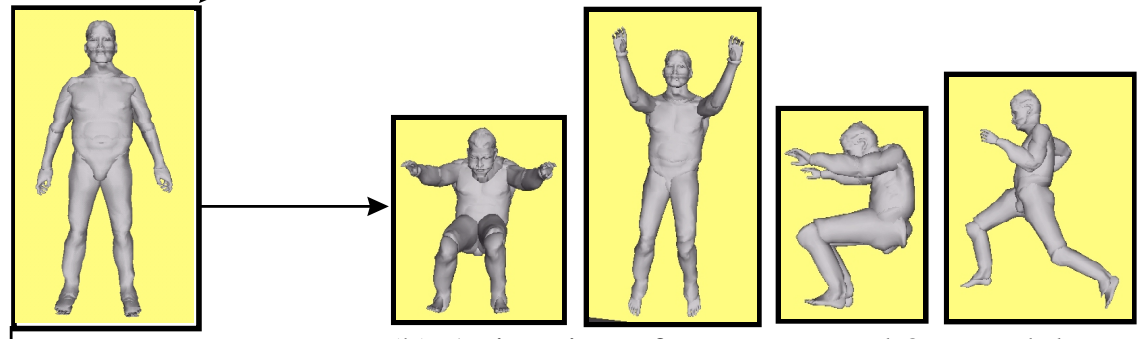

(g) 3D Model

(h) Animation of reconstructed 3D model

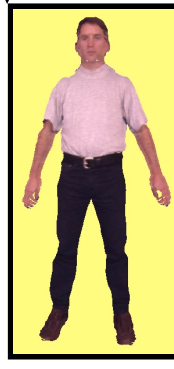

(i) Colour 3D model
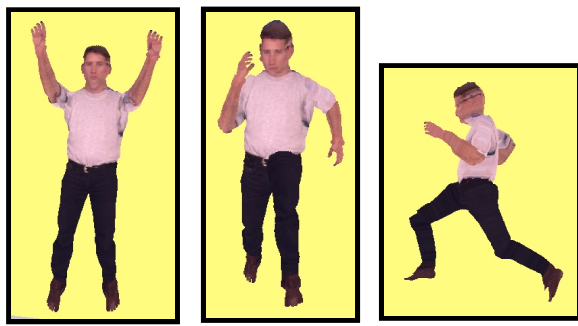

(j) Animation of reconstructed 3D colour model

Figure 1: Overview of model recdAstruction for an individual person 
is modelled by attaching either a $3 \mathrm{D}$ polygonal mesh segment to the joint for each body part or a single seamless polygonal mesh surface for the whole-body. For example the articulated structure of an arm can be represented by three joints shoulder-elbow-wrist and the shape by segments attached to each joint upper-arm-forearm-hand. The shape segments can be specified with multiple levels-of-detail to achieve both efficient and realistic humanoid animation. Material properties and texture maps can be attached to each body segment for rendering the model.

The model-based reconstruction algorithm introduced in this paper can use any reasonable generic humanoid body as the initial model which is modified to approximate the shape and texture of a particular person. The reconstruction algorithm can also handle models with multiple levels-of-detail for each body part. All reconstruction results presented in this paper are based on a publicly available humanoid model which is compliant with the draft standard and gives a reasonable compromise between representation quality and animation efficiency. The joint structure for the generic humanoid model consists of fifteen joints as illustrated in Figure 2(a). The model shape consists of fifteen body segments with a total of 10K mesh vertices and 20K triangular polygons. The rendered surface model is shown in Figure 2(b). The VRML97 specification allows movement animations based on interpolation of joint angles to be specified independent of the humanoid geometry. Figure2(c) and (d) show snap-shots of running and jumping animations.

The following nomenclature is used in later sections to refer to the polygonal model and associated texture map. Throughout this work the notation $\vec{x}=(x, y, z)$ refers to a $3 \mathrm{D}$ vector such as a mesh vertex. For each body part the polygonal mesh is specified as a list of $N_{v} 3 \mathrm{D}$ vertices, $\left\{\vec{v}_{i}=\left(x_{i}, y_{i}, z_{i}\right)\right\}_{i=1}^{N_{v}}$, and a list of $N_{t}$ polygons, $\left\{t_{r}=\left(\vec{v}_{i}, \vec{v}_{j}, \vec{v}_{k}\right)\right\}_{i=1}^{N_{t}}$. An image or texture map 2D coordinate is defined as $\vec{u}=(u, v)$ where $u$ is the vertical coordinate and $v$ is the horizontal coordinate with $u, v \in[0,1]$ and the origin at the top left-hand corner of the image. Texture mapping of an image onto a polygonal mesh is specified by a $2 \mathrm{D}$ texture coordinate for each mesh vertex, $\left\{\vec{u}_{i}=\left(u_{i}, v_{i}\right)\right\}_{i=1}^{N_{v}}$. 


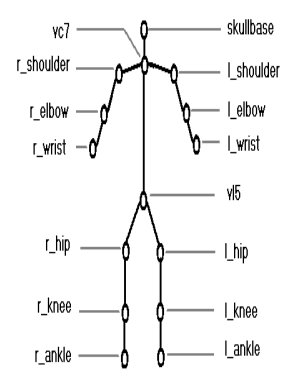

(a)Joints

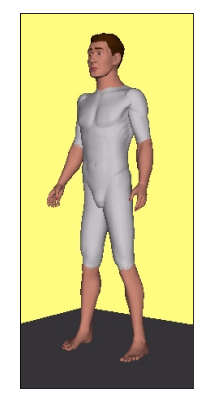

(b)Surface

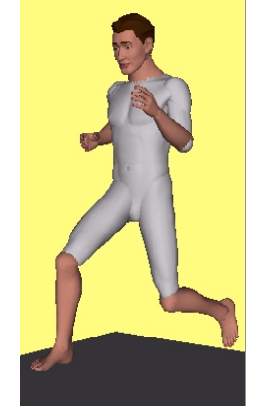

(a)Run

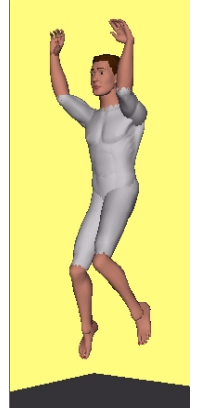

(b)Jump

Figure 2: Generic humanoid model based on the VRML draft specification

\subsection{Image capture, silhouette and feature extraction}

\subsubsection{Image capture}

An experimental system has been setup to capture whole body images of an individual from four orthogonal views (front,left,back,right). The four view camera configuration is illustrated in Figure 3(a). Colour images are captured using a Sony DXC-930P 3CCD camera with $756 \times 582$ picture elements. This gives a resolution of approximately $40 \times 40$ pixels for the subjects face. Images are taken against a photo-reflective blue screen backdrop called 'Truematte' [Popkin, 1997] which allows reliable foreground/background separation with arbitrary foreground lighting and clothing colours including most shades of blue. The subject stands in a standard pose similar to the generic model pose as shown in Figure 5(a). Currently each view is taken with a single camera with the subject rotating to present the required view to the camera. The use of a single camera may result in small changes of pose between views but has the advantage of identical intrinsic camera projection parameter for each view. The capture process results in a set of four data images, $I_{i}^{D} i=1 \ldots 4$, for orthogonal views of a specific person. Only two orthogonal views are required to approximate the 3D shape of a particular person. The use

of four views gives additional high-quality texture information for the front, back and sides of the body.

To model the image capture process we assume a pin-hole camera without lens distortion. The camera $3 \mathrm{D}$ to $2 \mathrm{D}$ projection can be expressed in homogeneous coordinates as:

$$
\overrightarrow{u^{\prime}}{ }_{i}=P_{i} \overrightarrow{x^{\prime}}=M E_{i} \overrightarrow{x^{\prime}}
$$


view. The use of a single camera avoids the requirement for accurate multi-camera calibration to register images from different views.

Intrinsic camera calibration is based on estimation of the camera parameters from direct measurement of the field-of-view for a plane orthogonal to the view direction. From the size of the horizontal and vertical field-of-view at a distance of three meters from the camera we estimate the camera focal lengths in image pixels as: $f_{u}=$ verticalpixels $\times \frac{\text { distance-to-camera }}{\text { vertical-height }} \approx$ $756 \frac{3}{2}=1134$ and $f_{v}=$ horizontalpixels $\times \frac{\text { distance-to-camera }}{\text { horizontal-height }} \approx 582 \frac{3}{1.5}=1044$. This gives an approximate estimate of the camera focal length. As all views of a person are taken with the same camera this estimate is sufficient for view integration and accurate camera calibration is not necessary. The image origin is taken as the center of the image which in pixel coordinates is $\left(o_{u}, o_{v}\right)=(378,291)$

There is no calibration for extrinsic camera parameters. The person is assumed to rotate approximately $90^{\circ}$ between front, left, back and right views. Extrinsic parameters $E_{i}$ are taken as orthogonal rigid-body transforms of the camera center at a distance of three meters from the origin of the world coordinate system as illustrated in Figure 3(a).

The approximate calibrated camera model $P_{i}=M E_{i}$ is used to generate a set of four synthetic images of the generic humanoid model, $I_{i}^{M} i=1 \ldots 4$. This is achieved by projection of each vertex $\vec{v}_{i}$ on the generic model to its corresponding image coordinates $\vec{u}_{i}$ using equation 1 .

A four-camera capture system could be used to capture all views simultaneously. This would have the advantage of avoiding movement of the person between views. A multi-camera system would require accurate camera calibration to enable view integration and considerably more space to setup.

\subsubsection{Silhouette extraction}

Silhouette extraction aims to construct the chain of image pixels which lie on the boundary between the image of the person and the background. A standard chroma-key technique is used to identify background pixels based on the percentage of blue in each pixel. Given an image pixel with red, green and blue colour components $(r, g, b)$ the percentage blue is $p_{b}=$ $100 \times(b /(r+g+b))$. A constant chroma-key threshold (50\%) is used together with an intensity threshold $(|b|>150)$ to reliably generate a binary image with each pixel labelled as either 
foreground or background. An example of an extracted binary silhouette image is shown in Figure 5(b).

The silhouette curve, $C_{i}^{D}$, for each captured image, $I_{i}^{D}$, is extracted by following an 8connected chain of pixels on the border of the foreground image of a person. We denote the silhouette by the chain of $N_{p} 8$-connected adjacent pixel coordinates $C_{i}^{D}=\left\{\vec{u}_{j}\right\}_{j=1}^{N_{P}}$ which is specified in counter-clockwise order with-respect-to the image view direction. An example of an extracted silhouette chain is shown in Figure 5(c). A similar process is performed on the synthetic images $I_{i}^{M}$ of the generic model using a binary threshold to obtain a set of model silhouette curves, $C_{i}^{M}$.

\subsubsection{Feature extraction}

The objective of feature extraction is to establish the correct correspondence between the captured data and synthetic model images for body parts such as the arms, legs, head and torso. Correct correspondence is essential for realistic animation of the reconstructed model of a person based on the articulated joint structure of the generic model. We therefore require robust extraction of a set of feature points for a wide range of changes in body shape, size and clothing. To achieve this we constrain the person to stand approximately in a pre-specified pose and wear clothing which allows both the arm pits and crotch to be visible such as a shirt and trousers. Given these assumptions an algorithm has been developed for reliable extraction and localisation of a set of features based on our knowledge of the silhouette contour structure.

The algorithm for extracting feature points is presented in Figure 4. Initially the algorithm traverses the silhouette contour $C_{i}$ to locate five extremum points, $\vec{u}_{e 1-e 5}$, on the contour. These correspond to the head, hands and feet as illustrated in Figure5(c). The extrema points can be reliably extracted for all silhouettes but their location varies significantly due to variation in shape and pose. Therefore, the extrema are used to identify five key feature points, $\vec{u}_{f 1-f 5}$, which can be accurately located even with large changes in shape and pose. The feature points correspond to the crotch, arm-pits and shoulders as shown in Figure 5(d).

This procedure gives reliable extraction of a set of key feature points for a wide range of people shape, size, clothing and hair-styles. It has been found that other potential features points such as the neck cannot be reliably localised as small changes in shape can result in a large variation in position. Resulting in a poor quality correspondence between the captured and 
generic model images. The set of extracted features are sufficient to accurately align the major body parts for a captured image silhouette (head, torso, arms, legs) with those of the generic model image. A similar procedure is applied for the side views to identify the tip of the nose as the left or right extremum on the head. Other body parts such as the fingers cannot be reliably identified with the image resolution used as each finger is less than three pixels across. Higher resolution images may permit correspondences to be established between additional body parts.

\subsubsection{Pose estimation}

Pose estimation identifies the angle of the arms and legs for a set of captured images of a specific person. This information is used to adjust the pose of the generic model to that of a particular individual. The pose of the arms, legs and head are estimated by computing the principal axis for the contour points corresponding to each of these components. If the set of contour points for a particular body part is $C_{i}=\left\{\vec{u}_{j}\right\}_{j=r}^{s}$ then the principal axis is given by:

$$
\vec{u}_{a x i s}=\frac{1}{(s-r+1)} \sum_{j=r}^{s} \vec{u}_{j}^{2}-\left(\frac{1}{(s-r+1)} \sum_{j=r}^{s} \vec{u}_{i}\right)^{2}
$$

The angle of the principal axis with the vertical gives the approximate pose of the body parallel to the image plane. The body part pose is used in the mapping to correct for small variations between the generic model and the captured image set for a particular individual.

\subsection{D-to-2D Silhouette Mapping}

The objective of mapping between the generic humanoid model and the captured images is to establish a dense correspondence for mapping each model part. Dense correspondence establishes a unique one-to-one mapping between any point, $\vec{u}^{M}$, inside the generic model silhouette and a point on the same body part, $\vec{u}^{D}$, inside the captured image silhouette. This correspondence is used to modify the shape of the generic humanoid model to approximate the shape of a particular individual. For example to achieve realistic arm movement for the reconstructed model of an individual it is necessary to map the projection of the arm on the generic model image to the corresponding arm on the captured image.

Body-part correspondence is established using the feature points, $u_{f 1-f 5}$, on the silhouette contours of the generic model and the captured data images. These features can be used to establish a correct correspondence for each part of the human body. Based on the five key 
1. Find the extremum points $u_{e 1-e 5}$ :

(a) Find the extremum point on the top of the head, $u_{e 1}$, as the contour point with minimum vertical coordinate, $u$ :

$u_{e 1}=\min \left(\left\{u_{j}\right\}_{j=1}^{N_{P}}\right)$ and $v_{e 1}=v_{j}$

(b) Find the extremum point on the left, $\vec{u}_{e 2}$, and right, $\vec{u}_{e 5}$, hands as the contour points with minimum and maximum horizontal coordinate, $v$ :

$$
\begin{aligned}
& v_{e 2}=\min \left(\left\{v_{j}\right\}_{j=1}^{N_{P}}\right) \text { and } u_{e 2}=u_{j} \\
& u_{e 5}=\max \left(\left\{v_{j}\right\}_{j=1}^{N_{P}}\right) \text { and } u_{e 5}=u_{j}
\end{aligned}
$$

(c) Evaluate the centroid of the silhouette contour:

$\vec{u}_{C}=\frac{1}{N_{P}} \sum_{j=1}^{N_{P}} \vec{u}(i)$.

(d) Find the extremum points on the left, $\vec{u}_{e 3}$, and right, $\vec{u}_{e 4}$, feet as the contour points with maximum vertical coordinate, $u$, either side of the centroids horizontal coordinate, $v_{C}$ :

$$
\begin{aligned}
& u_{e 3}=\max \left(\left\{u_{j}\right\}_{j=1}^{N_{P}}\right) \text { and } v_{e 3}=v_{j} \leq v_{C} \\
& u_{e 4}=\max \left(\left\{u_{j}\right\}_{j=1}^{N_{P}}\right) \text { and } v_{e 4}=v_{j} \geq v_{C}
\end{aligned}
$$

2. Find the feature points $u_{f 1-f 5}$ :

(a) Locate the key feature points corresponding to the crotch, $\vec{u}_{f 1}$, and the left, $\vec{u}_{f 2}$, and right, $\vec{u}_{f 3}$, arm-pits as the contour points with minimum vertical coordinate, $u$, which are between the corresponding hand and feet extremum points:

$\operatorname{crotch} u_{f 1}=\min \left(\left\{u_{j}\right\}_{j=e 3}^{e 4}\right)$ and $v_{f 1}=v_{j}$

left-armpit $u_{f 2}=\min \left(\left\{u_{j}\right\}_{j=e 2}^{e 3}\right)$ and $v_{f 2}=v_{j}$

right-armpit $u_{f 3}=\min \left(\left\{u_{j}\right\}_{j=e 4}^{e 5}\right)$ and $v_{f 2}=v_{j}$

(b) Locate feature points on the left, $\vec{u}_{f 4}$, and right, $\vec{u}_{f 5}$, shoulders with the same horizontal coordinate, $v$, as the arm-pit features $u_{f 2}$ and $u_{f 3}$ :

left-shoulder $u_{f 4}=\min \left(\left\{u_{j}\right\}_{j=e 1}^{e 2}\right)$ and $v_{f 4}=v_{f 2}$

right-shoulder $u_{f 5}=\min \left(\left\{u_{j}\right\}_{j=e 5}^{e 1}\right)$ and $v_{f 5}=v_{f 3}$

Figure 4: Algorithm for feature extraction from a silhouette contour $C_{i}$ for front and back views 


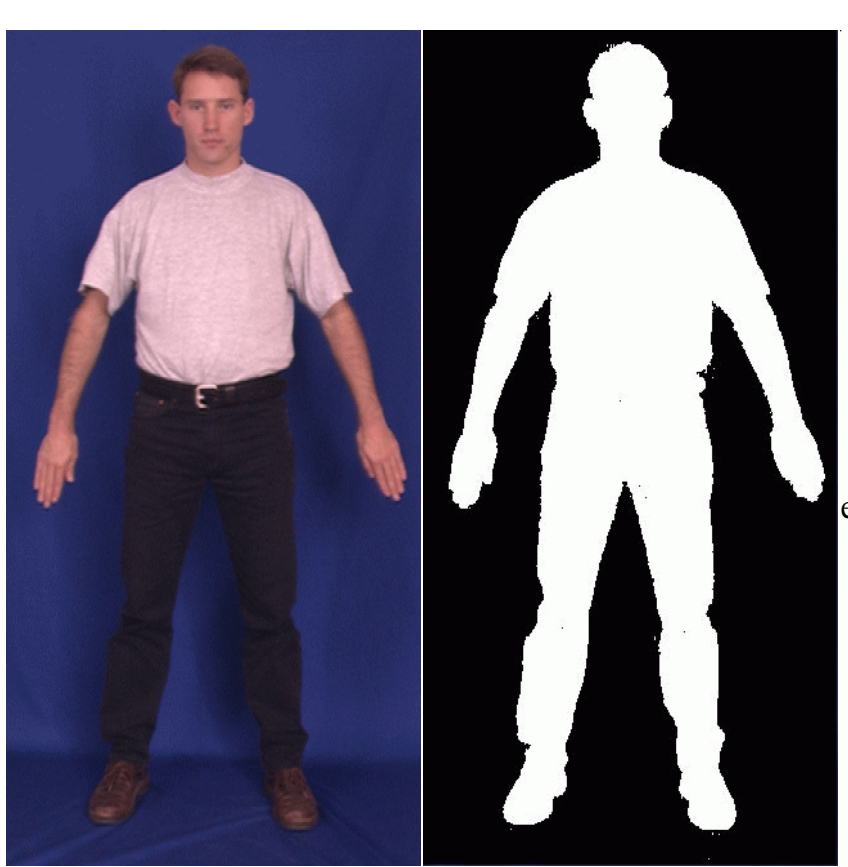

(a) Image

(b) Silhouette

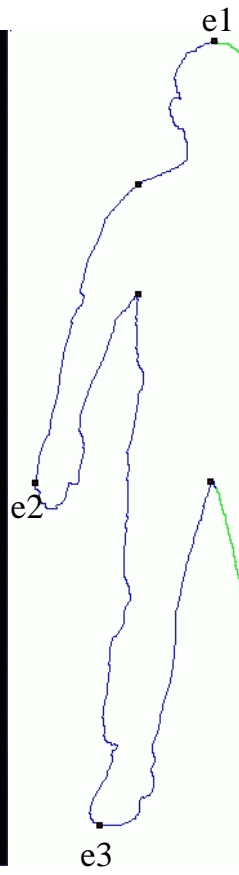

(c) Extrema

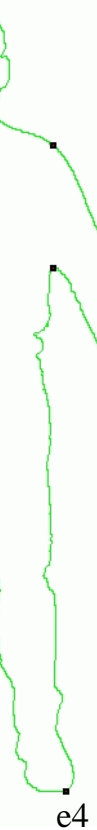

e4

(d) Features

Figure 5: Silhouette and feature extraction for front and side view silhouettes

points the human model is separated into seven functional parts as illustrated in Figure 6(a): head(1); shoulders(2); left-arm(3); right-arm(4); torso(5); left leg(6); right leg(7). Separating the silhouette images into body-part allows a dense mapping to be established independently for points inside each body-part silhouette.

A unique one-to-one correspondence between points inside the model and data sets for a particular body-part is established by a 2D linear mapping based on the relative dimensions of the silhouette. This is equivalent to a $2 \mathrm{D}$ affine transform in the image plane (rotation, scale, shear and translation). The mapping between corresponding points inside the silhouette for a particular body part is given as follows in homogeneous coordinates:

$$
\begin{gathered}
{\overrightarrow{u^{\prime}}}^{D}=S{\overrightarrow{u^{\prime}}}^{M} \\
S=\left[\begin{array}{lll}
s_{u} & s_{u v} & t_{u} \\
s_{v u} & s_{v} & t_{v} \\
0 & 0 & 1
\end{array}\right]
\end{gathered}
$$

The components $s$ represent the rotation, shear and scale between the parts and $t$ translation between body parts. If the Principal axis of the body parts are aligned $s_{u v}=s_{v u}=0$ and $s_{u}, s_{v}$ 
represent the horizontal and vertical scale factors respectively. The vertical scale factor, $s_{u}$, and translation, $t_{u}$, for a particular body part can be computed from the vertical limits, $\left[u_{\min }, u_{\max }\right]$. Similarly the horizontal scale factor, $s_{v}$ and translation $t_{v}$ are given by the horizontal limits, $\left[v_{\min }, v_{\max }\right]$, for a horizontal slice $(u=$ const) through the silhouette contour. The vertical and horizontal scale factors and translations are given by:

$$
\begin{aligned}
s_{u} & =\frac{u_{\max }^{D}-u_{\min }^{D}}{u_{\max }^{M}-u_{\min }^{M}} \\
t_{u} & =-s_{u} u_{\min }^{M}+u_{\min }^{D} \\
s_{v}(u) & =\frac{v_{\max }^{D}(u)-v_{\min }^{D}(u)}{v_{\max }^{M}(u)-v_{\min }^{M}(u)} \\
t_{v}(u) & =-s_{v}(u) v_{\min }^{M}(u)+v_{\min }^{D}(u)
\end{aligned}
$$

This mapping enables us to evaluate a unique one-to-one correspondence of points inside the data silhouette $\vec{u}_{D}$ for any point inside the model silhouette $\vec{u}_{M}$. This allows 2D information such as the colour from the captured model to be mapped to the silhouette of the generic model as illustrated in Figure 1 (f). The mapping achieves an exact correspondence at the feature points and a continuous mapping elsewhere including across boundaries between different body parts. The change in position $\Delta \vec{u}$ between model and data silhouette is given by:

$$
\vec{u}_{D}=\vec{u}_{M}+\Delta \vec{u}
$$

This 2D change in position in the image plane for a particular view can be used to estimate the change in position of a $3 \mathrm{D}$ point orthogonal to the view direction.

\subsection{D-to-3D Shape Mapping from Orthogonal Views}

The objective of the 2D-to-3D mapping is to combine the dense 2D-to-2D mapping information, $\Delta \vec{u}_{i}$, from multiple views, $i=1, \ldots, 4$ to estimate the 3D displacement, $\Delta \vec{x}$ of a point $\vec{x}$ on the surface of the 3D model. We first outline the theory for 2D-to-3D inverse projection and then present the application of this to our model-based reconstruction.

\subsubsection{D-to-3D Inverse Projection}

A point in the 2D camera image plane corresponds to an infinite ray in 3D space. Therefore, inverting the camera projection equation 1 gives the equation of a line in 3D space. From 


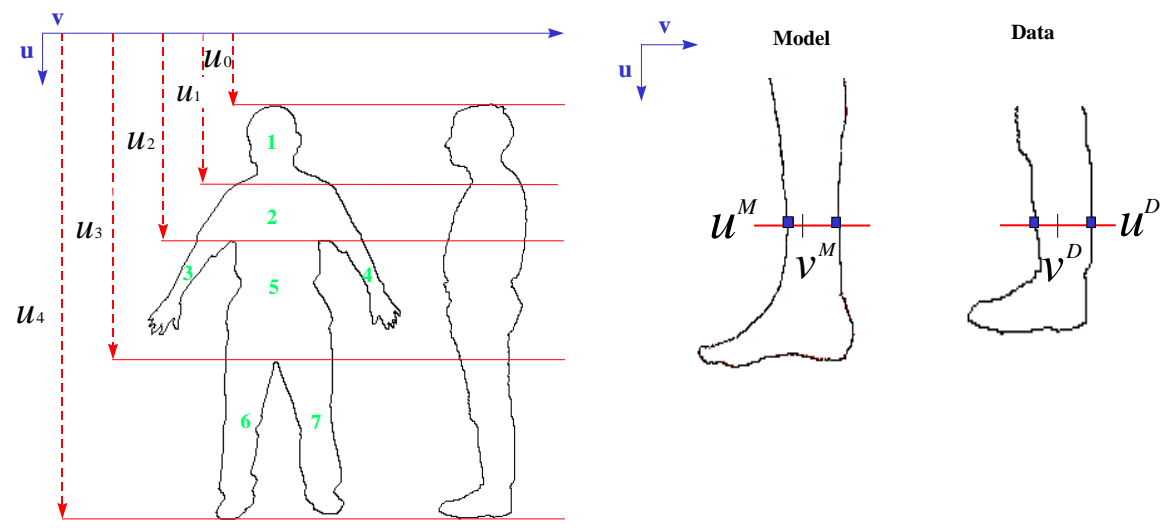

(a) Vertical mapping $\quad$ (b) Horizontal mapping

Figure 6: 2D-to-2D mapping between model and data silhouette

equation 1 the inverse projection in homogeneous coordinates for the $i^{\text {th }}$ view direction is:

$$
\begin{aligned}
{\overrightarrow{x^{\prime}}}^{D}={\overrightarrow{x^{\prime}}}^{M}+\Delta x_{i} & =\lambda_{i}\left(\vec{x}^{D}\right) P_{i}^{-1}\left({\overrightarrow{u^{\prime}}}_{i}^{M}+\delta{\overrightarrow{u^{\prime}}}_{i}\right) \\
& =\lambda_{i}\left(\vec{x}^{D}\right) E_{i}^{-1} M^{-1}\left({\overrightarrow{u^{\prime}}}_{i}^{M}+\Delta{\overrightarrow{u^{\prime}}}_{i}\right)
\end{aligned}
$$

where $\lambda_{i}$ is a scale factor equal to the orthogonal distance of the 3D point from the camera: $\lambda_{i}\left(\vec{x}^{D}\right)=\left\|R_{i} \vec{x}^{D}+\vec{t}_{i}\right\|$. The estimated 3D displacement component $\Delta x_{i}$ is orthogonal to the camera view direction $\vec{n}_{i}$. The inverse camera calibration and transform matrices are given by:

$$
\begin{aligned}
M^{-1} & =\left[\begin{array}{ccc}
\frac{1}{f_{u}} & 0 & -\frac{o_{u}}{f_{u}} \\
0 & \frac{1}{f_{v}} & -\frac{o_{v}}{f_{v}} \\
0 & 0 & 1 \\
0 & 0 & 0
\end{array}\right] \\
E_{i}^{-1} & =\left[\begin{array}{ll}
R_{i}^{-1} & -R_{i}^{-1} \vec{t}_{i} \\
\overrightarrow{0}^{T} & 1
\end{array}\right]
\end{aligned}
$$

Thus the 3D point on the model in real coordinates $\vec{x}^{D}$ is on the 3D line represented by: 


$$
\vec{x}^{D}=\vec{x}^{M}+\Delta \vec{x}_{i}=\lambda_{i}\left(\vec{x}^{D}\right) R_{i}^{-1} M^{-1}\left(\vec{u}_{i}^{M}+\Delta \vec{u}_{i}\right)-R_{i}^{-1} \vec{t}_{i}
$$

Equation 6 defines the relationship between a line through the real 3D point, $\vec{x}^{D}$, and the estimated 2D displacement for the $\Delta \vec{u}$ in the image plane for the corresponding point on the generic model $\vec{x}^{M}$. As the real 3D point, $\vec{x}^{D}$, is unknown we must estimate the scale factor $\lambda_{i}\left(\vec{x}^{D}\right)$ in order to estimate the 3D displacement, $\Delta \vec{x}$, orthogonal to the camera view direction.

\subsubsection{Single view 3D displacement}

The 2D-to-2D mapping for the $i^{t h}$ view gives an estimate of the displacement of a 3D point $\vec{x}$ between the projection of the generic model $\vec{u}_{i}^{M}$ and the projection of the surface of a real person $\vec{u}_{i}^{D}$. This $2 \mathrm{D}$ image plane displacement, $\Delta \vec{u}_{i}$, defined by equation 4 , can be used to estimate the 3D displacement component, $\Delta \vec{x}_{i}=\left(\Delta x_{i}, \Delta y_{i}, \Delta z_{i}\right)$, of the projected 3D point, $\vec{x}$, on the generic model orthogonal to the $i^{\text {th }}$ image view direction. This is achieved by estimating the inverse projection of the displacement of the $2 \mathrm{D}$ point $\Delta \vec{u}_{i}$ in the camera image. The inverse projection can be estimated uniquely from our knowledge of the distance to the corresponding 3D point, $\vec{x}^{M}$, on the generic model. This approximates the unknown distance to the corresponding 3D point, $\vec{x}^{D}$, on the captured person which we want to estimate. From equation 6 we obtain the approximate 3D displacement as:

$$
\Delta \vec{x}_{i} \approx \lambda_{i}\left(\vec{x}^{M}\right) R_{i}^{-1} M^{-1}\left(\vec{u}_{i}^{M}+\Delta \vec{u}_{i}\right)-R_{i}^{-1} \vec{t}_{i}-\vec{x}^{M}
$$

where we have approximated the distance along the ray by the distance to the corresponding point on the generic model: $\lambda_{i}\left(\vec{x}^{D}\right) \approx \lambda_{i}\left(\vec{x}^{M}\right)=\left\|R_{i} \vec{x}^{D}+\vec{t}_{i}\right\|$. The distance to the generic model is a reasonable approximation as the distance between the camera and person, $(\approx 3 m)$, is large relative to the difference in 3D surface position, $(\approx 0.1 m)$, between the model and person. Section 6.2 presents an analysis of the errors in the reconstructed model based on this assumption.

Estimation of the 3D displacement component orthogonal to the view direction is illustrated in Figure 7(a). A single view image $I_{i}^{D}$ gives an approximation of the component of 3D displacement $\Delta \vec{x}_{i}$ of a known 3D point $\vec{x}^{M}$ on the generic model orthogonal to the $i^{\text {th }}$ view direction $\vec{n}_{i}=\left(n_{x}, n_{y}, n_{z}\right)$ such that $n_{i} \bullet \Delta \vec{x}_{i}=0$. For example for the front image the view direction, $\vec{n}_{0}=(0,0,1)$, is aligned with the z-axis of the world-coordinate system consequently 
the displacement of an image point $\Delta \vec{u}_{0}=\left(\Delta u_{0}, \Delta v_{0}\right)$ gives an approximation of the $3 \mathrm{D}$ displacement orthogonal to the view direction $\Delta \vec{x}_{0}=\left(\Delta x_{0}, \Delta y_{0}, 0\right)$. Similarly a left side view with view direction $\vec{n}_{1}=(1,0,0)$ gives an estimate of the $3 \mathrm{D}$ displacement $\Delta \vec{x}_{1}=\left(0, \Delta y_{1}, \Delta z_{1}\right)$.

Applying the estimated displacement component to each vertex on the $3 \mathrm{D}$ generic model results in an affine transform of the 3D model orthogonal to the view direction. Application of the 2D-to-3D mapping for a single view is illustrated in Figure 8(a). Reprojecting the modified model results in a silhouette which approximates the captured silhouette shape. The surface shape for $3 \mathrm{D}$ points whose projection is inside the silhouette is a $2 \mathrm{D}$ affine transform of the shape of the generic model.

\subsubsection{Multi-view 3D displacement}

Combining the estimated displacement components from two or more orthogonal views of a point $\vec{x}$ we obtain an estimate of the 3D displacement $\Delta \vec{x}$, as illustrated in Figure 7(b). Only two orthogonal views are required to estimate all three components of the displacement where each view gives estimates of two of the three components. To obtain an estimate of the 3D displacement from two or more views we can either take estimates of each displacement component from a single view or combine the estimates. Displacement components from multiple views can be combined by averaging to estimate the 3D displacement:

$$
\Delta \vec{x}=\left[\begin{array}{c}
\frac{1}{N_{x}} \sum_{i=0}^{N_{x}} \Delta x_{i} \\
\frac{1}{N_{y}} \sum_{i=0}^{N_{y}} \Delta y_{i} \\
\frac{1}{N_{z}} \sum_{i=0}^{N_{z}} \Delta z_{i}
\end{array}\right]
$$

where $N_{x}, N_{y}$ and $N_{z}$ are the number of displacement estimates in a particular direction. Combining displacement components from multiple views averages out any misalignment or change in shape of the 2D silhouettes for different views. This gives an estimate of the 3D displacement $\Delta \vec{x}$ of a point on the generic model $\vec{x}^{M}$. The estimated displacement is used to approximate the 3D shape of a specific person:

$$
\vec{x}^{D}=\vec{x}^{M}+\Delta \vec{x}
$$

The generic model shape is modified by estimating the 3D displacement $\Delta \vec{x}\left(\vec{v}_{j}\right)$ for each vertex $\vec{v}_{j}$. The model vertex position, $\vec{v}_{j}$, is projected to the $2 \mathrm{D}$ image plane using the camera 


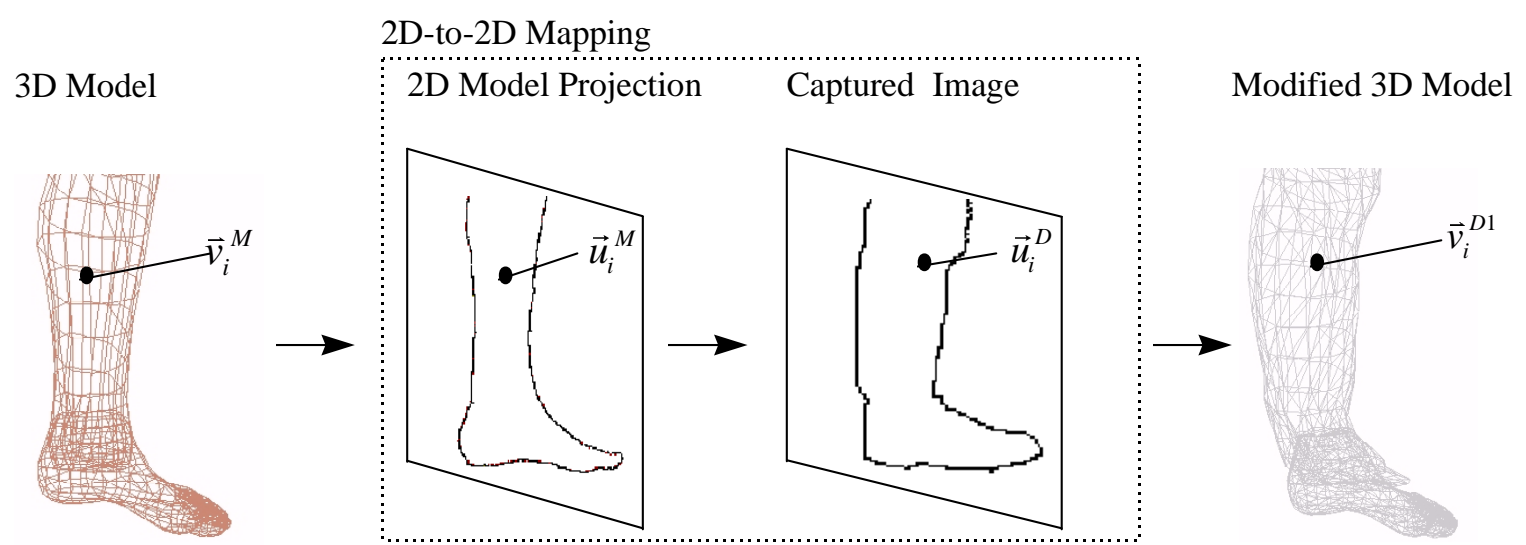

(a) Mapping for a single view

Cross-section of 3D Model

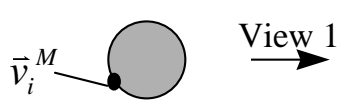

Model Projection Slice
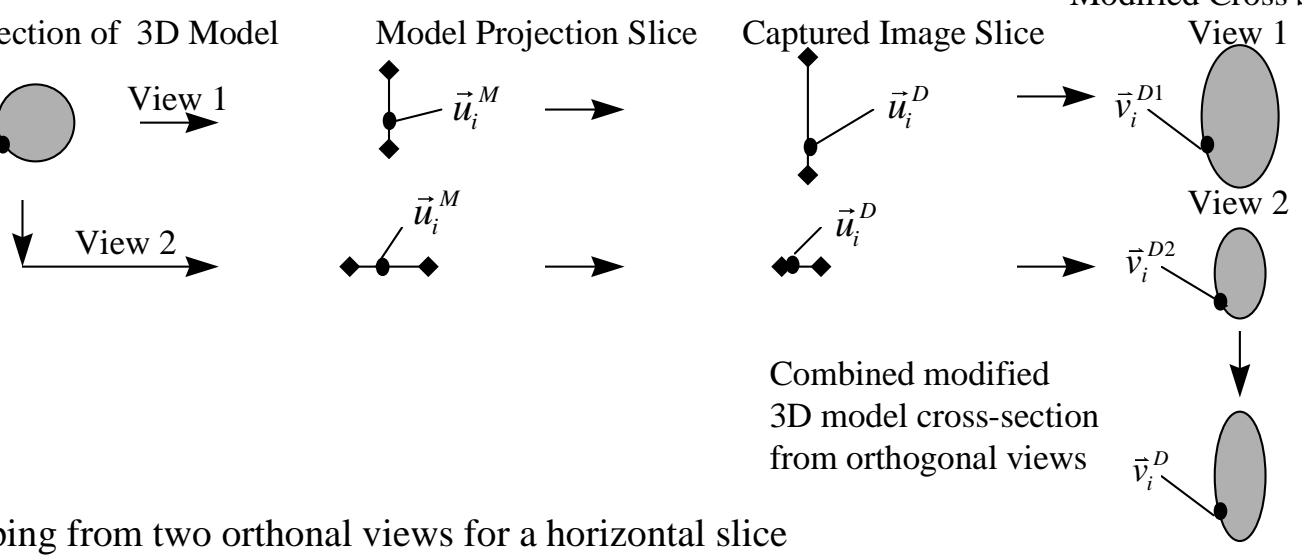

Modified Cross Section

(b) Mapping from two orthonal views for a horizontal slice

Figure 8: 2D-to-3D mapping between model and data silhouette for orthogonal views 


\subsection{Colour texture mapping}

The 2D-to-2D mapping for a single view enables the colour texture of the captured data image to be mapped onto the projected model image as illustrated in Figure 1(f). For all points, $\vec{u}^{M}$, inside the projected generic model we know the corresponding point on the captured data image $\vec{u}^{D}$. To texture map the 3D model we project each vertex $\vec{v}_{i}^{M}$ to obtain the image coordinates $\vec{u}_{i}^{M}$ and from the 2D-to-2D mapping obtain the corresponding data image coordinates $\vec{u}_{i}^{D}$.

The problem is then to obtain a single texture map for each body part by integrating the four view images. Integration of multiple view images to obtain a single texture map has previously been addressed for face [Akimoto et al., 1993, Mortlock et al., 1997, Lee and Magnenat-Thalmann, 1998 and object [Niem and Wingebermuhle, 1997, Sannier and Magnenat Thalmann, 1997] modelling by back-projecting to a single cylindrical texture map. This assumes that the model geometry is known accurately such that corresponding points from different images project to the same point on the model and consequently back project to the same point on the cylindrical texture map. In our case this assumption is not valid as the model geometry is not known accurately and the image correspondences between views are unknown. However, we have found that using the approximate geometric shape information from the reconstructed model, together with the mapping for each view onto the generic model, we can achieve visually realistic models using a cylindrical texture mapping approach. The mapping information for each body part approximately aligns the multi-view images. Texture blending is performed to achieve a smooth transition for overlapping images which are not precisely aligned.

Cylindrical texture mapping is used as for the majority of body parts (arms, legs and torso) it results in a unique one-to-one (injective) projection from the model surface to the texture map. The resulting cylindrical texture can then be stored in a 2D image format. However, for regions of complex geometry, such as the ears and hair, multiple points on the model surface may project to the same point on the cylinder. This prevents a unique texture coordinate being derived for each point on the model and may result in visual artifacts in the texture mapped model. In this work we use a cylindrical texture map for all body parts. The cylindrical texture

coordinates $\vec{\phi}_{i}=\left(\theta_{i}, h_{i}\right)$ for a vertex on the reconstructed data model $\vec{v}_{i}^{D}=\left(x_{i}^{D}, y_{i}^{D}, z_{i}^{D}\right)$ is evaluated as:

$$
\vec{\phi}_{i}=\left(\theta_{i}, h_{i}\right)=\left(\tan ^{-1}\left(\frac{\left(z_{i}^{D}-c_{z}\right)}{\left(x_{i}^{D}-c_{x}\right)}\right), y_{i}^{D}\right)
$$




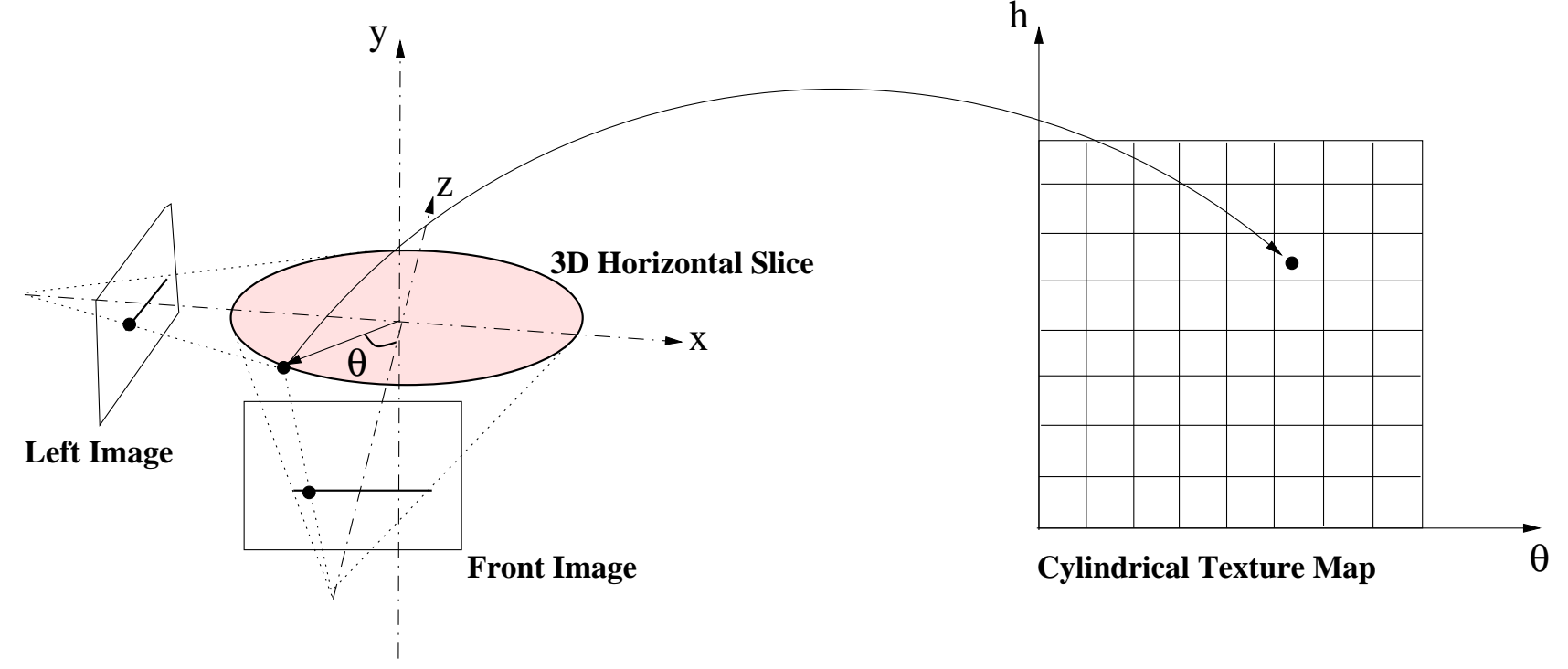

Figure 9: Cylindrical texture mapping of a point on a slice through the 3D model

Where the model center is given by the center of the silhouette slices for the front and side view as: $c_{x}=\left(x_{\max }-x_{\min }\right) / 2$ and $c_{z}=\left(z_{\max }-z_{\min }\right) / 2$. The cylindrical mapping for a horizontal slice through the model is illustrated in Figure 9. This mapping is used to evaluate a cylindrical texture coordinate $\vec{\phi}_{i}$ for all mesh verticies on the $\vec{v}_{i}^{D}$ on the reconstructed model.

This enables us to map the colour information from the captured images into the cylindrical texture image. For each mesh vertex $\vec{v}_{i}^{D}$ we have a cylindrical texture coordinate $\vec{\phi}_{i}$ and image coordinate in two orthogonal view images $\vec{u}_{i}^{D}$. Therefore, for each triangle we can map the colour information from the captured images to the cylindrical texture map. As in previous work [Mortlock et al., 1997] barycentric coordinates of points inside the triangle are used to map the captured image colour for points inside the triangle to pixels in the cylindrical texture map.

Mapping from captured image to cylindrical coordinates results in overlapping regions between orthogonal view images. To obtain a smooth transition between overlapping textures a ramp weighting function is used to integrate colour information. For example in cylindrical coordinates the front image maps in the range $\left[\frac{\pi}{2}, \frac{3 \pi}{2}\right]$ and the left image $[0, \pi]$. To integrate the views we map colour values from the front view only for $\left[\frac{3 \pi}{4}, \frac{5 \pi}{4}\right]$ and from left view only for $\left[\frac{5 \pi}{12}, \frac{7 \pi}{12}\right]$. In the region $\left[\frac{7 \pi}{12}, \frac{3 \pi}{4}\right]$ we integrate the front $\vec{r}_{f}$ and left $\vec{r}_{l}$ colour values according to the ramp weighting function: $\vec{r}=(1-w) \vec{r}_{f}+w \vec{r}_{l}$ where $w$ varies linearly in the range [0,1] over the interval and $\vec{r}=(r, g, b)$ is the red, green, blue component colour vector. This simple 


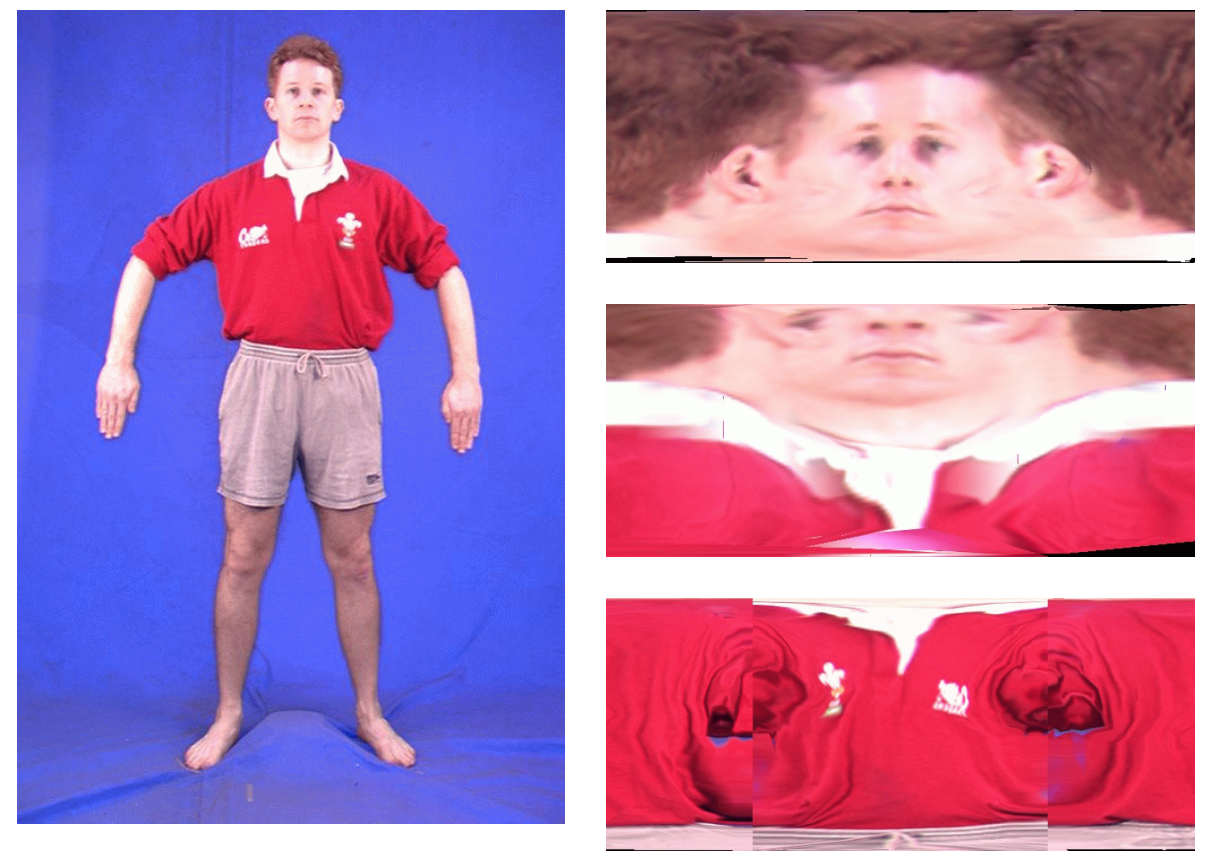

Figure 10: Original image and integrated texture maps for head, shoulders and torso

linear integration algorithm has been found to produce reasonable results and therefore a more sophisticated blending algorithm has not been used.

This approach generates a single cylindrical texture map for each body part by integrating information from the four views. Figure 10 shows examples of integrated texture maps obtained for several body parts. All four views are integrated for the head and shoulder texture maps. For the torso only the front and back images are used as the sides are not visible resulting in a discontinuity in the image texture.

\section{Results}

The experimental system for whole-body modelling presented in this paper has been used to capture models of approximately twenty individuals wearing a variety of clothing. Images are captured using a single camera with the person turning to present their front, left, back and right sides. The results presented in this section show models generated automatically from the captured images without any manual intervention using the approach introduced in section 4. 


\subsection{Whole-body modelling}

The model-based reconstruction algorithm has been used to capture models of approximately twenty individuals wearing a variety of clothing. Subjects were constrained to wear clothing such as trousers and shirt which allows the location of the arm pits and crotch to be visible in the front view. The silhouette extraction and feature point identification algorithms presented in section 4 were applied to automatically identify the five key points. The feature point extraction algorithm was found to give valid feature point labelling for all captured images. This includes people with a wide variety of clothing including baggy shirts and skirts which obscure the physical location of the crotch and arm-pits. The feature points enable the body to be segmented into seven parts. The model-based reconstruction from silhouettes is applied to all body parts except the hands and feet. Hands and feet are modelled by scaling the corresponding part of the generic model as there is insufficient information on the silhouette images to identify feature points.

Reconstructed 3D models for three individuals are shown in Figure 5.1(a). The left-hand column shows the original front and left side colour photos. The second column shows the estimated joint positions and reconstructed model front view silhouette. from the front view. The remaining columns show the 3D model with and without texture and a single frame from a running animation. These results demonstrate that the automatic reconstruction generates a recognisable 3D facsimile of the real person. The reprojected silhouette shape of the person for the four views matches the silhouette shape of the captured image. It should be noted that the silhouette shape for the hands and feet does not match as these are only scaled versions of the generic model hands and feet.

The reconstructed 3D whole-body shape based on the silhouette data gives a good approximation of the shape for a particular individual. Some artifacts can be seen in the shape between the arm-hand and leg-foot. This is due to to poor segmentation of the feet from the legs and hands as no feature points are available. The 3D shape approximation is of sufficient accuracy to give a recognisable model when texture mapped with the image colour information. The joint positions estimated from the silhouette shape give an approximation of the anatomical structure of a person. Provide the clothing does not obscure the true crotch and arm-pit locations the estimated joint positions are sufficiently accurate to enable realistic animation.

Further examples of reconstructed models for male and female subjects are presented in 
Figure 5.1(b). Note that one of the subjects is wearing a long skirt which violates the assumption that the crotch point is visible. In this situation a good model of the persons shape and appearance is generated. However, the joint positions are incorrect and result in an invalid animation as the body-part correspondence between the generic model and person are not correct. Anatomical constraints could be used to give improved estimates of the joint position in this situation. Figure 5.1(c) shows reconstructions for the same person wearing different clothing. This example demonstrates that the approach can be used to generate a set of models for a particular individual suitable for multiple virtual world applications (business,sports,leisure).

These results demonstrate the feasibility of obtaining realistic whole-body models from sets of captured images. The quality of the final texture mapped 3D models is limited by the resolution of the captured images. The current resolution is sufficient for realistic approximation when viewing the whole body but not for close-ups of individual parts such as the face. Highresolution images of individual body parts would allow reconstruction of realistic models for close-up views.

Currently the principal limitation of the reconstruction from silhouettes is the quality of the face models generated. Due to the absence of feature point labelling on the face the alignment with the generic model is inaccurate. This may result in bumps in the face shape and distortion of the face shape in the presence of long hair which obscures the sides of the face. Previous feature based approaches to face modelling [Lee and Magnenat-Thalmann, 1998, Mortlock et al., 1997] could be used to improve face modelling if full resolution face images were captured. However, current techniques for face modelling may also fail to automatically reconstruct face shape in the presence of hair and glasses.

\subsection{Animation}

Animation of the reconstructed 3D models for particular individuals performing standard movements is illustrated in Figure 12. In this work avatars have been animated using simple animation sequences for walking, running and jumping which are pre-defined from captured motion data. The animation sequence in the form of joint angle/axis rotations are applied directly to the virtual model for a particular individual. Multiple virtual people models can be run in real-time with reasonably smooth movement on a $400 \mathrm{MHz}$ Pentium platform.

Figure 12(a) shows a simple virtual catwalk scene with several animated virtual people. 

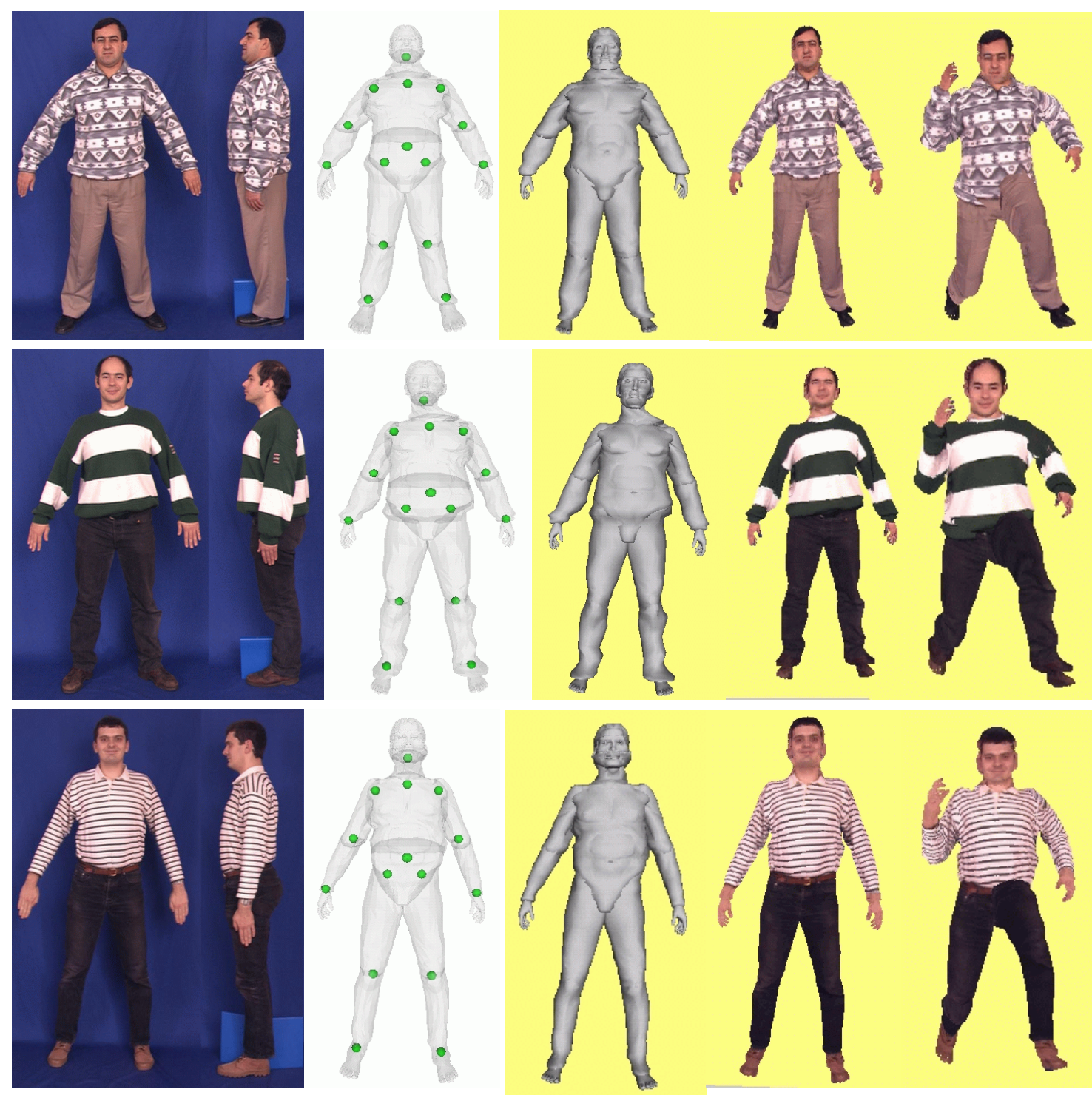

(a) Reconstructed models: Photo; Joints; 3D Model; 3D Colour Model; Running Animation

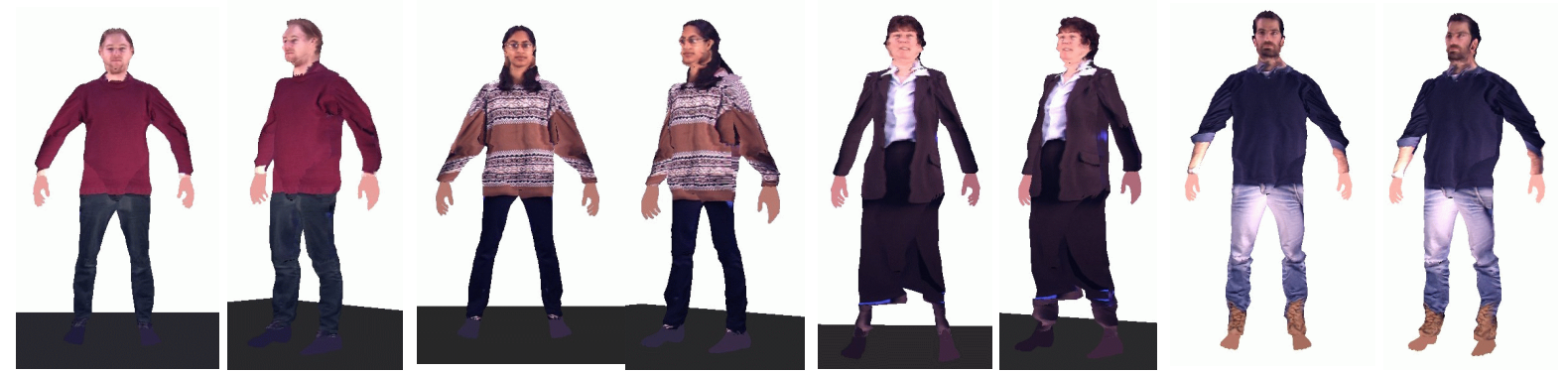

(b) Examples of male/female virtual people
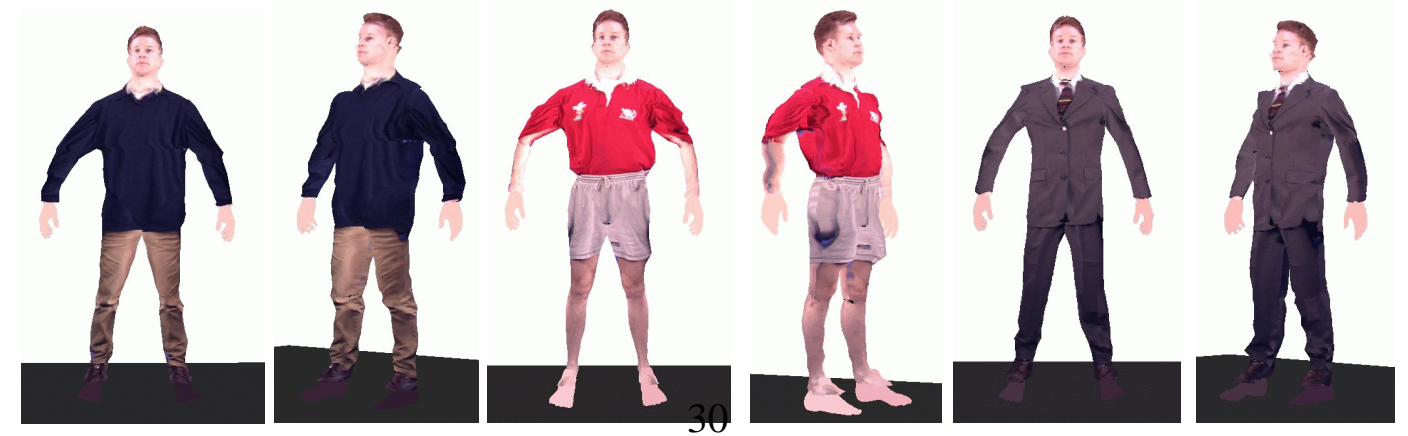

(c) Models of the same person with different clothing

Figure 11: Reconstructed 3D texture mapped models for individual people 
The articulated structure of the generic humanoid model is modified for a particular individual by mapping the $3 \mathrm{D}$ joint positions using the 2D-to-3D mapping algorithm presented in the previous section. The animation parameters based on joint angle interpolation are the same as for the generic model. Animation of movements such as walking, running and jumping using a common set of parameters results in reasonable movements of a particular individual for VR applications. However, the animation is based on a rigid 3D model which does not incorporate changes in the body or clothing shape during movement. This results in visible artifacts in the humanoid animation.

Figure 12(b) shows a sequence from the walking animation for a particular individual. As the clothing texture is rigidly fixed to the segments of the generic model the clothing moves rigidly with the body parts resulting in visual artifacts as regions of the texture appear and disappear. This can be seen in the pelvis region for the front views of Figure 12(b). A second visual artifact in the animation occurs due to the segment based structure of the generic model. The reconstruction algorithm modifies each segment in the body to approximate the silhouette shape for a particular person. When the model is animated the deformed segments may no longer form a smooth surface resulting in visible gaps near the joints. This can be seen for the knee from the side view of the animation sequence of Figure 12(b).

The reconstructed joint structure and 3D colour models achieve realistic animation of a persons movement. However, visual artifacts occur due to the generic model structure and the simplicity of the animation. Greater realism could be achieved using a more sophisticated humanoid model based on a seamless mesh which can be deformed in real-time [Kalra and Magnenat Thalm Physics-based models could also be used to achieve realistic deformation of the skin and clothing. However, this may be prohibitively expensive for real-time applications.

\subsection{Avatar Booth}

A commercial booth system developed using the approach presented in this paper is illustrated in Figure 13. The booth uses a single camera capture system with the person turning to present the four front, back and side views. Manual interaction is used to identify key feature points on the face. The use of manually identified face feature points ensures that faces are correctly reconstructed when the silhouette is obscured by the persons hair. Whole-body models are then generated automatically using the approach presented in this paper. A generic humanoid model 


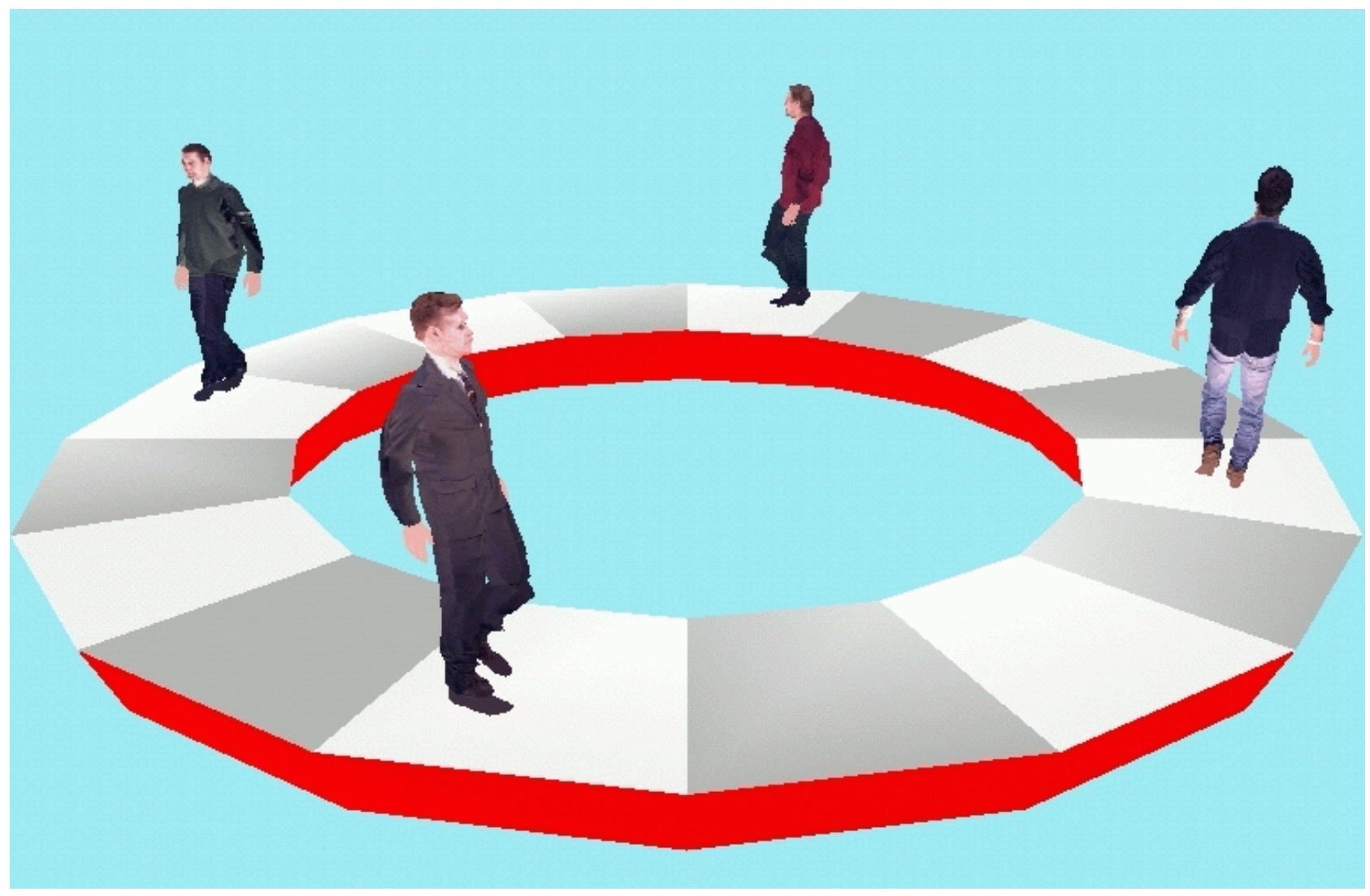

(a) A simple catwalk scene with four models

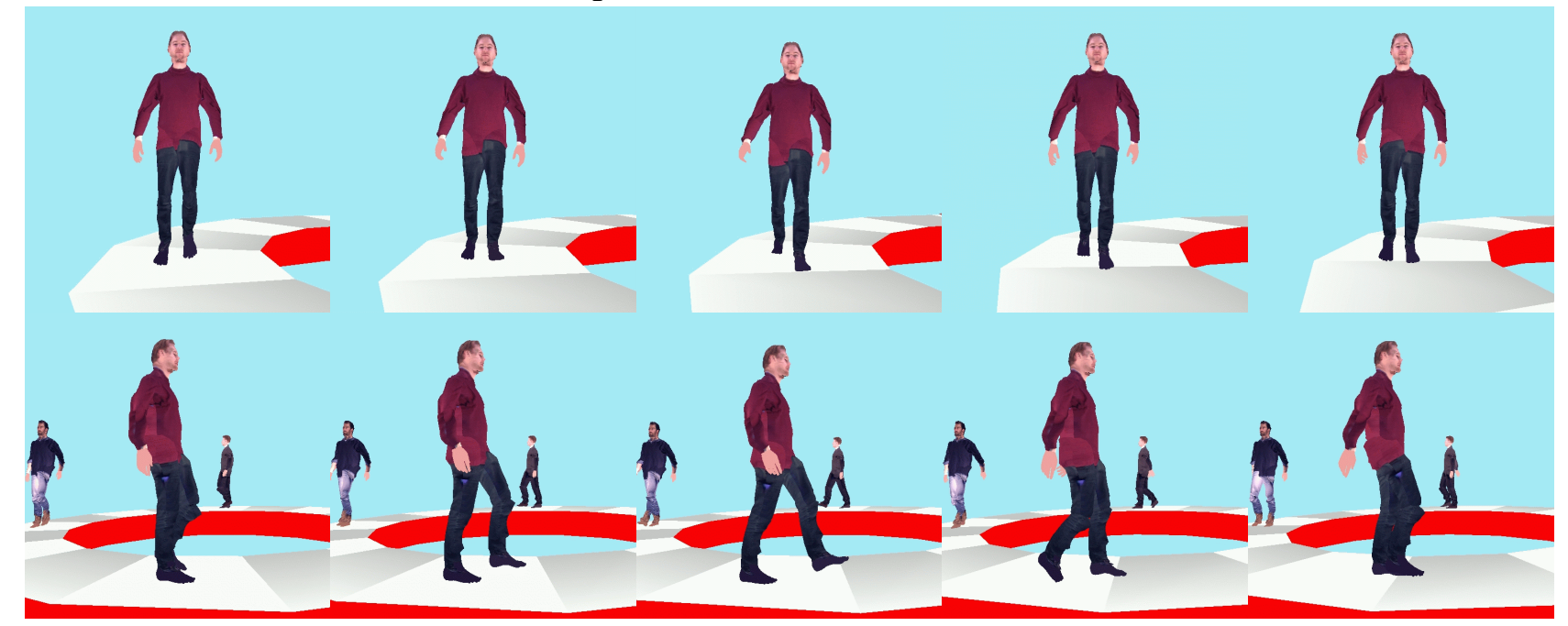

(b) Front and side views of the walking animation

Figure 12: Virtual people in a virtual catwalk scene animation 


\section{The person is standing in an approximately known pose.}

2. The person is wearing clothing which allows the arm-pits and crotch to be identified.

3. The persons shape can be approximated by an affine transform of the generic humanoid from two or more orthogonal views.

4. The anatomical joint structure of a person can be approximated by an affine transform of the joint structure for the generic model.

5. The estimated shape information is sufficiently accurate to enable integration of the captured images into a single texture map.

The first two assumptions are satisfied by constraining the clothing and pose of the person captured. These assumptions are satisfied for a wide range of people size, shape and clothing. The results demonstrate that the reconstruction is insensitive to a wide variation in the initial pose. If the constraints on clothing are not satisfied the persons shape is correctly reconstructed but their estimated joint positions may be incorrect.

The results demonstrate that the approximation of the body shape from the orthogonal view silhouettes gives a reasonable model of the body surface. This is the case for all body parts except the head, hands and feet. For other body parts non-uniform scaling of the generic model shape gives a good approximation of the shape of a particular individual due to their inherent symmetry. Similarly, non-uniform scaling of the joint structure gives a reasonable approximation of the joint positions. The results demonstrate that the approximate shape is sufficiently accurate to enable blending of overlapping images from multiple views to obtain a single texture map. Texture mapping of a model with approximately the same shape as a particular person gives a realistic virtual model.

An additional assumption made in the single camera capture system used in this work is that the approximate intrinsic and extrinsic camera calibration enables satisfactory reconstruction. The shape approximation error resulting from the intrinsic calibration is discussed in section 6.2. The extrinsic calibration is determined by the person turning by $90^{\circ}$ to present each view. The reconstruction has been found to be insensitive to errors in both the intrinsic and extrinsic calibration. No reduction in the visual quality of the reconstructed model has been observed due to errors in the calibration. 


\subsection{Shape Approximation Error}

The model-based reconstruction of 3D shape from silhouettes presented in section 4.4 is based on the assumption that the distance to a point on a particular individual $z^{D}$ is approximately equal to the distance to the corresponding point on the generic model $z^{M}$. This assumption is used to enable evaluation of the inverse projection given by equation 5 . In practice the error in this distance is the principal factor contributing to the error in the estimation of the $3 \mathrm{D}$ silhouette shape for a particular person.

Given an estimate of the maximum error in the distance between the camera and the person we can estimate the error in the 3D silhouette shape. This is illustrated in Figure 14(a). For a pin-hole camera aligned with the world coordinate system we have the relationships: $f_{u}=$ $x^{M} / z^{M}=x^{D} / z^{D}$ and $f_{v}=y^{M} / z^{M}=y^{D} / z^{D}$. Now if we know that $z^{D}=z^{M} \pm \epsilon_{z}$ then we can derive the following relationship:

$$
\epsilon_{x}=\frac{\epsilon_{z}}{z^{M}} x^{M} \quad \epsilon_{y}=\frac{\epsilon_{y}}{z^{M}} y^{M}
$$

For the current experimental setup, as illustrated in Figure 3, the distance between the camera and person is approximately $z^{M}=3 m$ and the maximum error in a persons position is $\epsilon_{z}= \pm 0.1 \mathrm{~m}$. From equation 11 the resulting maximum percentage error in the estimated 3D silhouette width or height is approximately $3 \%$. For a typical persons width of $0.6 \mathrm{~m}$ the corresponding error in the estimated point on the $3 \mathrm{D}$ silhouette is $\epsilon_{x}= \pm 0.01 \mathrm{~m}$ and the resulting error in the estimated width of the person is $\pm 0.02 \mathrm{~m}$.

This level of accuracy is adequate for the proposed application of capturing human models to represent people in virtual worlds and games. However, this accuracy is not sufficient for clothing applications or anthropometric measurement where a maximum error of less than $1 \%$ $(0.01 \mathrm{~m})$ is required. Current laser based whole-body measurement systems are more appropriate for this application domain.

The above analysis gives an approximate maximum error for points on the $3 \mathrm{D}$ model silhouette. The reconstructed surface shape approximation of a particular individual is based on the shape of the generic humanoid model. The shape of the generic humanoid model is scaled according to a linear affine transform to fit the 3D silhouette shape of a particular individual as discussed in section 4. A particular individual with clothing may exhibit large shape variations 


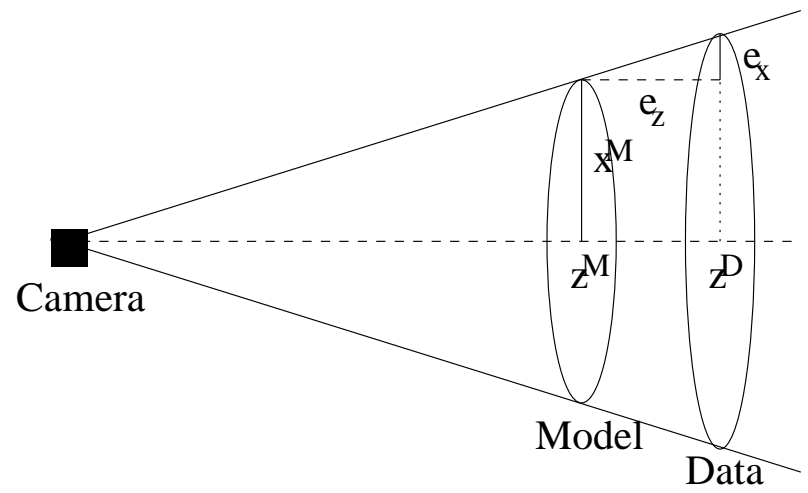

(a) Errors in 3D silhouette due to error in distance to person

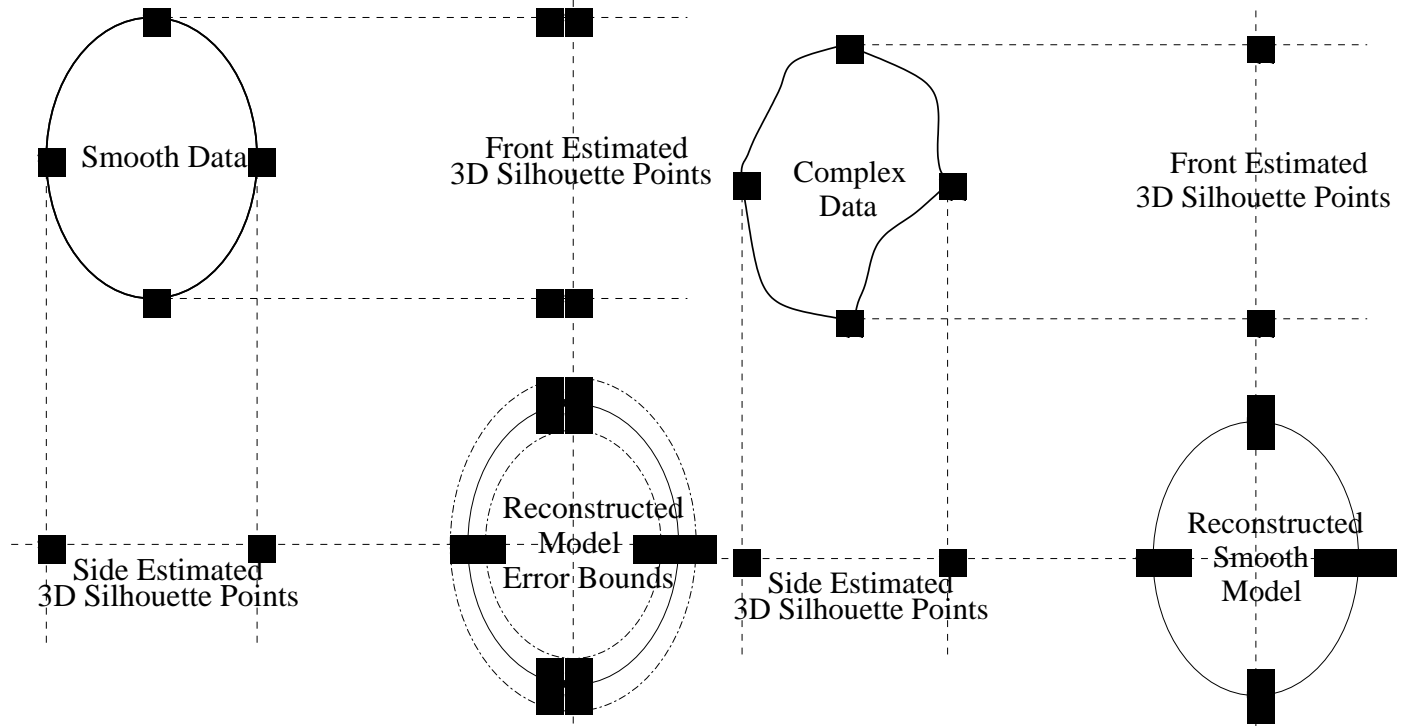

(b) Smooth surface data

(c) Complex surface data

Figure 14: Errors in 3D silhouette estimation and model-based surface reconstruction

which are not captured in the orthogonal view 3D silhouettes. Therefore errors which are larger than the maximum silhouette error may occur particularly for complex body parts such as the head with long hair. For approximately smooth symmetrical body parts such as the arms, legs and torso transforming the model to fit the orthogonal view silhouettes will give an approximation error for the body surface similar to the error in the estimated 3D silhouettes. Hence, for smooth body parts we can expect the maximum error to be of the same order of magnitude as the error in the silhouette approximation. The surface approximation error based on scaling the generic model is illustrated in Figure 14(b) and (c) for a smooth surface shape and a complex shape.

A limited quantitative evaluation of the accuracy of the 3D silhouette and model-based surface reconstruction has been performed. This evaluation was performed by direct comparisons 
of measurements of the dimensions of a person with the corresponding dimensions on the reconstructed 3D model. Results of this analysis indicate that the error in the 3D silhouette dimensions is of the order of $\pm 0.01 \mathrm{~m}$ for most of the body excluding the face, hands and feet. Similarly, for smooth body parts such as the arms, legs and torso the error in the estimated surface can be estimated by taking cross-sections through the reconstructed mode. From this analysis it has been found that in general the error is less than $\pm 0.03 m$ of the real body shape for smooth body parts with approximately the same shape as the original model. The sample of models taken for this evaluation is limited to those illustrated in this paper. Full evaluation of the accuracy of the reconstruction process could be achieved by comparison of the reconstructed models with measurements of the same subject acquired using a whole-body laser measurement system. This has not been performed due to the lack of access to such a device. However, as the focus of the proposed approach is not on measurement of whole-body shape it is considered that this analysis is non essential to validate the current approach. Reconstructed models should be evaluated qualitatively in terms of visual appearance.

\subsection{Computational Cost}

The primary cost in the reconstruction algorithm is the computation of displacements for mesh vertices on the generic model to approximate the silhouette shape of the captured data images. This requires first projecting each mesh vertex on the generic model to the $2 \mathrm{D}$ image plane using equation 1 . The projection matrix can be pre-computed hence this operation requires 14 multiplication and 12 addition operations. The next stage is to map the $2 \mathrm{D}$ vertex projection from the model to data silhouette. The 2D-to-2D silhouette mapping is pre-computed as a lookup table which maps each horizontal slice on the model silhouette to the corresponding slice on the data silhouette. The lookup table contains the pre-computed horizontal and vertical scale factors for each horizontal slice. This lookup requires a single operation per vertex. Hence, the cost of 2D-to-2D mapping for each vertex is determined by the cost of implementing equation 3 which requires two multiplications and two addition operations per vertex. Finally, the 2D data vertex position is reprojected into 3D space using equation 5. The inverse projection matrix is pre-computed. Evaluation of the inverse projection for a single view direction requires 16 multiplication and addition operations per vertex. This sequence of operations is repeated for each vertex in each view direction. Hence, the computational complexity of the reconstruction 
algorithm is $O\left(N_{v}\right)$ where $N_{v}$ is the number of mesh verticies. The number total of operations per vertex for reconstruction from four views is 128 floating point multiplications and 120 additions.

The experimental implementation on a $400 \mathrm{MHz}$ PentiumII PC Platform requires approximately 3 minutes to perform the complete reconstruction from image capture to texture mapped 3D model. This is for a generic humanoid model containing a total of $10 \mathrm{~K}$ mesh verticies. This process is completely automatic requiring no user intervention. It is envisaged that this computation time could be significantly reduced with further optimisation of the software. Commercial development of this approach has reduced the reconstruction time to less than 15 seconds on a similar platform.

\section{Conclusions}

A model-based approach has been introduced for automatic reconstruction of an articulated 3D colour model of a particular person from a set of colour images. Results demonstrated that this approach achieves recognisable models of individuals with a wide range of shape, size and clothing. The reconstructed model represents the 3D shape, colour texture and articulation structure required for animating movements. This approach enables low-cost capture of models of people in a VRML H-Anim avatar format suitable for populating virtual worlds. The principal contribution of this work are:

- Novel method for model-based reconstruction of shape from silhouettes.

- Whole-body reconstruction of shape and appearance from multi-view images.

- Capture of models for clothed people.

- Automatic reconstruction of full body animated models.

The results presented demonstrate the feasibility of model-based reconstruction of realistic representations of individual people from sets of images. However, several issues could be addressed in future work to achieve photo-realistic animated models:

- Facial feature point labelling for accurate modelling and animation [Mortlock et al., 1997, Lee and Magnenat-Thalmann, 1998]. 
- Capture of a person in multiple poses for accurate reconstruction of kinematic structure [Plankers et al., 1999, Kakadiaris and Metaxas, 1998a].

- Seamless deformable generic model for realistic animation.

- High-resolution image acquisition for improved photo-realism.

- Synchronous image acquisition from multiple views to avoid movement.

- Increased number of views to reduce occlusion.

- Multiple levels-of-detail to efficiently represent shape.

Further development of this system and integration with previous work on face and body modelling will give incremental improvements in the quality of the reconstructed models. The silhouette based approach for mapping a generic humanoid model to a particular person could be used to generate animated models from 3D whole-body surface measurements acquired from passive stereo [Plankers et al., 1999] or commercial laser-stripe scanners.

The results presented in this paper demonstrate the potential of a low-cost whole-body system for capturing recognisable 3D models of individual people from sets of colour images. Current models of people are suitable for representing an individual in a shared virtual environment, interactive games or generating animated image sequences from pre-defined motion.

\section{Acknowledgement}

This research was supported by the EPSRC, UK Funding Council on Advanced Fellowship AF/95/2531 held by Dr. Adrian Hilton and Grant GR/89518 'Functional Models: Building Realistic Models for Virtual Reality and Animation'.

\section{References}

[Akimoto et al., 1993] Akimoto, T., Suenaga, Y., and Wallace, R. (1993). Automatic creation of 3d facial models. IEEE Computer Graphics and Applications, 13(5):16-22.

[Blanz and Vetter, 1999] Blanz, V. and Vetter, T. (1999). A Morphable Model for the Synthesis of 3D Faces. In Proc. ACM SIGGRAPH, pages 187-194. 
[DeCarlo and Metaxas, 1996] DeCarlo, D. and Metaxas, D. (1996). The Integration of Optical Flow and Deformable Models with Applications to Human Face Shape and Motion Estimation. In Conference on Computer Vision and Pattern Recognition, pages 231-238.

[DeCarlo and Metaxas, 1999] DeCarlo, D. and Metaxas, D. (1999). Combining Information using Hard Constraints. In Conference on Computer Vision and Pattern Recognition, pages 132-138.

[DeCarlo et al., 1998] DeCarlo, D., Metaxas, D., and Stone, M. (1998). An Anthropometric Face Model using Variational Techniques. In Proc. ACM SIGGRAPH, pages 67-74.

[Dekker et al., 1998] Dekker, L., Khan, S., West, E., Buxton, B., and Treleaven, P. (1998). Models for understanding the $3 \mathrm{~d}$ human body form. In Proc.IEEE Workshop on ModelBased 3D Image Analysis, pages 65-74.

[Escher and Magnenat-Thalmann, 1997] Escher, M. and Magnenat-Thalmann, N. (1997). Automatic 3D Cloning and Real-Time Animation of a Human Face. In Proc. of IEEE International Conference on Computer Animation, Geneva, Switzerland, pages 58-66.

[Fitzgibbon et al., 1998] Fitzgibbon, A. W., Cross, G., and Zisserman, A. (1998). Automatic 3D model construction for turn-table sequences. In Koch, R. and Van Gool, L., editors, 3D Structure from Multiple Images of Large-Scale Environments, LNCS 1506, pages 155-170. Springer-Verlag.

[Fua, 1998] Fua, P. (1998). Face Models from Uncalibrated Video Sequences. In Modelling and Motion Capture Techniques for Virtual Environments - Magnenat-Thalmann,N. and Thalmann,D. (Eds.), pages 214-228. Lecture Notes in Artificial Intelligence 1537, Springer Verlag.

[Fua and Miccio, 2000] Fua, P. and Miccio, C. (2000). Animated Heads from Ordinary Images: A Least Squares Approach. Computer Vision and Image Understanding, (to appear).

[Geisen et al., 1997] Geisen, G., Mason, G., Houston, V., Taylor, C., and Graham, J. (1997). Automatic detection, identification and registration of anatomical landmarks. In Proc. $39^{\text {th }}$ Annual Meeting of the Hum.Fact. and Erg.Soc.(2), pages 750-753. 
[Gu et al., 1998] Gu, J., Chang, T., Mak, I., Gopalsamy, S., Shen, H., and Yuen, M. (1998). A 3D Reconstruction System for Human Body Modeling. In Proc. of Modelling and Motion Capture Techniques for Virtual Environments - Magnenat-Thalmann,N. and Thalmann,D. (Eds.), pages 229-240. Lecture Notes in Artificial Intelligence 1537, Springer Verlag.

[Guenter et al., 1998] Guenter, B., Grimm, C., Wood, D., Malvar, H., and Pighin, F. (1998). Making Faces. In Proc. ACM SIGGRAPH, pages 55-66.

[Hilton et al., 1999] Hilton, A., Beresford, D., Gentils, T., Smith, R., and Sun, W. (May 1999). Virtual people: Capturing human models to populate virtual worlds. In IEEE International Conference on Computer Animation, pages 174-185.

[Hilton and Goncalves, 1995] Hilton, A. and Goncalves, J. (1995). 3D scene representation using a deformable surface. In IEEE Workshop on Physics Based Modelling, pages 24-30. IEEE.

[Kakadiaris and Metaxas, 1998a] Kakadiaris, I. and Metaxas, D. (1998a). Three-dimensional human body model acquisition from multiple views. International Journal of Computer Vision, 30(3):191-218.

[Kakadiaris and Metaxas, 1998b] Kakadiaris, I. and Metaxas, D. (1998b). Vision-based animation of digital humans. In IEEE Computer Animation, pages 144-152.

[Kalra and Magnenat Thalmann, 1998] Kalra, N. and Magnenat Thalmann, N. (1998). Realtime animation of virtual humans. IEEE Computer Graphics and Applications, 18(5):4256.

[Kanade and Rander, 1997] Kanade, T. and Rander, P. (1997). Virtualized reality: Constructing virtual worlds from real scenes. IEEE MultiMedia, 4(2):34—47.

[Koenen, 1996] Koenen, R. e. (1996). Coding of Moving Pictures and Audio. http://drogo.cselt.stet.it/mpeg/standards/mpeg-4.htm.

[Kurihara and Kiyoshi, 1990] Kurihara, T. and Kiyoshi, A. (1990). A transformation method for modeling and animation of the human face from photographs. State of the Art In Computer Animation, Springer Verlag, pages 45-57. 
[Lee and Magnenat-Thalmann, 1998] Lee, W.-S. and Magnenat-Thalmann, N. (1998). Head Modeling from Pictures and Morphing in 3D with Image Metamorphosis Based on Triangulation. In Modelling and Motion Capture Techniques for Virtual Environments - MagnenatThalmann,N. and Thalmann,D. (Eds.), pages 254-268. Lecture Notes in Artificial Intelligence 1537, Springer Verlag.

[Lee et al., 1995] Lee, Y., Terzoupoulos, D., and Walters, K. (1995). Realistic modeling for facial animiation. In Proc. ACM SIGGRAPH, pages 55-62.

[Lejeune and Ferrie, 1996] Lejeune, A. and Ferrie, F. (1996). Finding the parts of objects in range images. Computer Vision and Image Understanding, 64(2):230-247.

[McInerney and Terzopoulos, 1996] McInerney, T. and Terzopoulos, D. (1996). Deformable models in medical image processing. Medical Image Analysis, 1(2):91-108.

[Moezzi et al., 1997] Moezzi, S., Tai, L.-C., and Gerard, P. (1997). Virtual view generation for 3d digital video. IEEE MultiMedia, 4(2):18-26.

[Mortlock et al., 1997] Mortlock, A., Machin, D., McConnell, S., and Sheppard, P. (1997). Virtual conferencing. BT Technology Journal, 15(4):120-129.

[Niem and Wingebermuhle, 1997] Niem, W. and Wingebermuhle, J. (1997). Automatic reconstruction of $3 \mathrm{~d}$ objects using a mobile monoscopic camera. In Proc. IEEE International Conferences on Recent Advances in 3D Digital Imaging and Modeling, Ottawa, Canada, pages $173-180$.

[Paquette, 1996] Paquette, S. (1996). 3D Scanning in Apparel Design and Human Engineering. IEEE Computer Graphics and Applications, 16(9):11-15.

[Pighin et al., 1998] Pighin, F., Hecker, J., Lischinski, D., Szeliski, R., and Salesin, D. (1998). Syntehsizing Realistic Facial Expressions From Photographs. In Proc. ACM SIGGRAPH, pages $75-84$.

[Plankers et al., 1999] Plankers, R., D’Apuzzo, N., and Fua, P. (1999). Automated body modeling from video sequences. In Proc. IEEE International Workshop on Modelling People, pages 45-53. IEEE Computer Society Press. 
[Popkin, 1997] Popkin, D. (1997). The Real Guide to Virtual Studios. BBC Resources.

[Roehl, 1997] Roehl, B. (1997). Draft Specification for a Standard VRML Humanoid, Version 1.0. http://ece.uwaterloo.ca/h-anim/.

[Sannier and Magnenat Thalmann, 1997] Sannier, G. and Magnenat Thalmann, N. (1997). A user-friendly texture-fitting methodolgy for virtual humans. In Proc. Computer Graphics International, pages 167-176.

[Szeliski, 1990] Szeliski, R. (1990). Rapid octree construction from image sequences. CVGIP: Image Understanding, 58(1):23-32.

[Terzopoulos, 1994] Terzopoulos, D. (1994). From physics-based representation to functional modeling of highly complex objects. In NSF-ARPA Workshop on Object Representation in Computer Vision, pages 347-359. Springer Verlag.

[Terzopoulos et al., 1988] Terzopoulos, D., Witkin, A., and Kass, K. (1988). Constraints on deformable models: Recovering 3d shape and nonrigid motion. Artificial Intelligence, 36(1):91-123.

[Wingbermuhle and Weik, 1997] Wingbermuhle, J. and Weik, S. (1997). Highly realistic modeling of persons for 3d videoconferencing systems. In IEEE Signal Processing Society 1997 Workshop on Multimedia Signal Processing. 


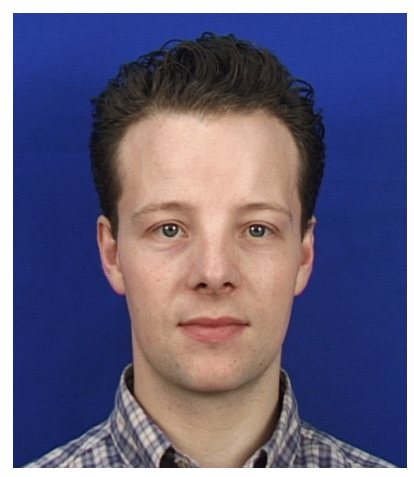

Hilton

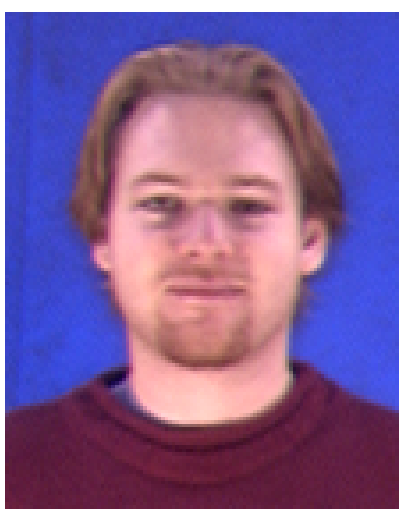

Smith

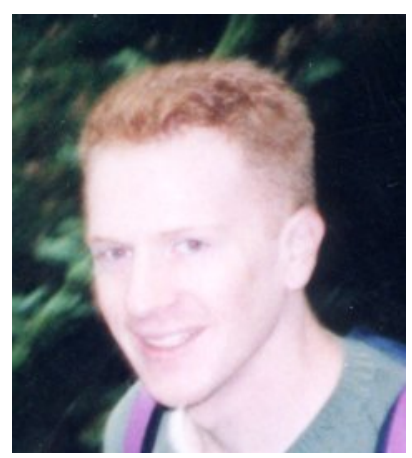

Beresford

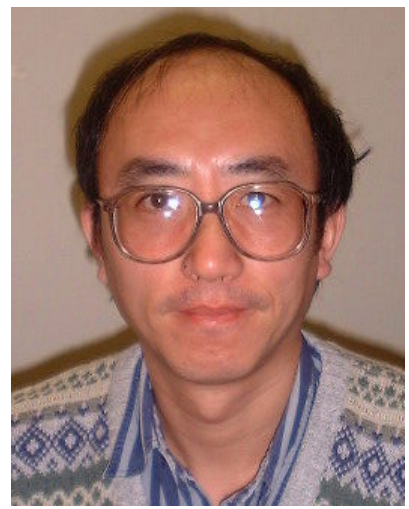

Sun

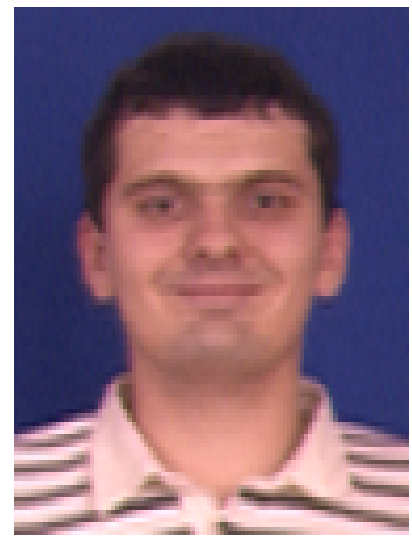

Gentils

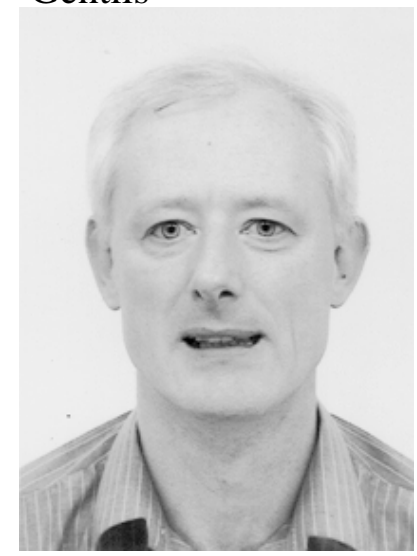

Illingworth

Dr. Adrian Hilton received a B.Sc.(Hons.) in Mechanical Engineering and a D.Phil. degree from the University of Sussex in 1988 and 1992 respectively. In 1992 he joined the CVSSP at the University of Surrey working on 3D shape capture for realistic computer graphics and accurate inspection. This work received Best Paper awards from the journals Pattern Recognition in 1996 and IEE Electronics and Communications in 1999. Collaboration with 3D Scanners Ltd. resulted in the first hand-held system for capturing 3D object models which was awarded a 1996 EU IT 'Grand Prize' for innovation. In 1997 he was awarded a five year EPSRC Advanced Fellowship and in 1999 became lecturer in 3D Digital Media and Broadcast. Research into 3D human modelling from digital photographs has been exploited by AvatarMe Ltd. to develop the first $3 \mathrm{D}$ booth for generating animated models of people. His active research interests include model-based 3D vision for capturing human movement and photo-realistic 3D models for computer animation.

Daniel Beresford graduated from The University of Reading in 1996 with a Bachelor of Engineering degree in Integrated Engineering. In 1997 he received a Master of Research degree 
from University College London. Since May 1998 he has been a Ph.D. student in the Computer Vision group at The University of Surrey. His research area is that of Human Body Modelling. Thomas Gentils graduated from the University of Orleans, France in 1999 with a Degree in Electronics and Signals. He was a visitor to the CVSSP at the University of Surrey for a period of five months in 1997 funded on the EU ERASMUS program as part of his professional training. During this period he worked with Adrian Hilton in initiating the research on modelling people from digital images.

Raymond Smith graduated from the University of Surrey in 1998 with a Master of Engineering degree in Information Systems Engineering. Since October 1998, he has been a Ph.D. Student in the Centre for Vision, Speech and Signal Processing at the University of Surrey, performing research into the automatic creation of 3D models suitable for animation. He also has interests in VRML, human modeling and animation.

Wei Sun is a research fellow in 3D-version group at University of Surrey. He received BSc and MSc in mathematics. His research areas are in computer animation and graphics since 1995. Professor John Illingworth is Professor of Machine Vision at Surrey University. He has a doctorate in Physics but has been active in research in computer vision, image processing and pattern recognition for the last 18 years. He has published over 130 papers in refereed journals and conferences. He is a Fellow of the Institution of Electrical Engineers and edits IEE Proceedings on Vision Image and Signal Processing. 
*Figure 1: Overview of model reconstruction for an individual person

Figure 2: Generic humanoid model based on the VRML draft specification

Figure 3: Image capture setup

Figure 4: Algorithm for feature extraction from a silhouette contour $C_{i}$ for front and back views

Figure 5:Silhouette and feature extraction for front and side view silhouettes Figure 6: 2D-to2D mapping between model and data silhouette

Figure 7: 3D Displacement estimation from multiple view 2D image displacements

Figure 8: 2D-to-3D mapping between model and data silhouette for orthogonal views

Figure9: Cylindrical texture mapping of a point on a slice through the 3D model

Figure 10: Original image and integrated texture maps for head, shoulders and torso

*Figure 11: Reconstructed 3D texture mapped models for individual people with animated movements

*Figure 12: Virtual people in a virtual catwalk scene animation

Figure 13:Proto-type avatar booth to populate virtual worlds

(courtesy AvatarMe Ltd. www.avatarme.com)

Figure 14: Errors in 3D silhouette estimation and model-based surface reconstruction

$(*=$ Reproduce in colour $)$ 